\title{
Gluino contribution to radiative $B$ decays: Organization of QCD corrections and leading order results
}

\author{
Francesca Borzumati, ${ }^{1,2}$ Christoph Greub, ${ }^{3}$ Tobias Hurth, ${ }^{1,4}$ and Daniel Wyler ${ }^{5}$ \\ ${ }^{1}$ Theory Division, CERN, CH-1211 Geneva 23, Switzerland \\ ${ }^{2}$ Scuola Internazionale Superiore di Studi Avanzati (SISSA), I-34013 Trieste, Italy \\ ${ }^{3}$ Institut für Theoretische Physik, Universität Bern, CH-3012 Bern, Switzerland \\ ${ }^{4}$ Max-Planck-Institut für Physik, Werner-Heisenberg-Institut, D-80805 Munich, Germany \\ ${ }^{5}$ Institut für Theoretische Physik, Universität Zürich, CH-8057 Zurich, Switzerland
}

(Received 11 November 1999; published 7 September 2000)

\begin{abstract}
The gluino-induced contributions to the decay $b \rightarrow s \gamma$ are investigated in supersymmetric frameworks with generic sources of flavor violation. It is shown that, when QCD corrections are taken into account, the relevant operator basis of the standard model effective Hamiltonian gets enlarged to contain (i) magnetic and chromomagnetic operators with a factor of $\alpha_{s}$ and weighted by a quark mass $m_{b}$ or $m_{c}$, (ii) magnetic and chromomagnetic operators of lower dimensionality, also containing $\alpha_{s}$, and (iii) four-quark operators weighted by a factor $\alpha_{s}^{2}$. Numerical results are given, showing the effects of the leading order QCD corrections on the inclusive branching ratio for $b \rightarrow s \gamma$. Constraints on supersymmetric sources of flavor violation are derived.
\end{abstract}

PACS number(s): 12.60.Jv, 12.38.Bx, 13.25.Hw

\section{INTRODUCTION}

Processes involving flavor changing neutral currents (FCNC's) provide invaluable guidelines for supersymmetric model building. The experimental measurements of the rates for these processes, or the upper limits set on them, impose in general a reduction of the large number and size of parameters in the soft supersymmetry-breaking terms present in these models. Among these processes, those involving transitions between first- and second-generation quarks, namely FCNC processes in the $K$ system, are considered as the most formidable tools to shape viable supersymmetric flavor models. Moreover, the tight experimental bounds on some flavordiagonal transitions, such as the electric dipole moment of the electron and of the neutron, as well as $g-2$, help constrain soft terms inducing chirality violations.

Several supersymmetric models have so far emerged, with specific solutions to the chiral-flavor problem. Among them are two classes of models in which the dynamics of flavor sets in above the supersymmetry breaking scale and in which the subsequent flavor problem is destroyed by the mechanisms of communicating supersymmetry breaking to the experimentally accessible sector. They are known as minimal supergravity models, MSUGRA, i.e. minimal supersymmetric standard models in which supergravity is the mediator between the supersymmetry-breaking sector and the visible sector [1], and gauge-mediated supersymmetry-breaking models (GMSBs) [2], in which the communication between the two sectors is realized by gauge interactions. In other classes of models, particular flavor symmetries are introduced, which link quarks and squarks: models in which an alignment of squarks and quarks is assumed [3], and models in which the solution to the flavor problem is obtained by advocating heavy first- and second-generation squarks [4-7]. In the latter, the splitting between squarks of first and second generation and those belonging to the third generation relies on a $U(2)$ flavor symmetry $[5,7]$.

Neutral flavor transitions involving third-generation quarks do not yet pose serious threats to these models. One exception comes from the decay $b \rightarrow s \gamma$, the least rare flavorand chirality-violating process in the $B$ system. It has been detected, but the precision of the experimental measurement of its rate is not very high at the moment. Nevertheless, this measurement already has the effect of carving out some regions in the space of free parameters of most of the models in the above classes (see for example [8]; for a recent analysis, see [9] and references therein). They also drastically constrain several somewhat tuned realizations of models in these classes $[10,11]$. Once the precision in the experimental measurement has increased, this decay will undoubtedly gain efficiency in selecting the viable regions of the parameter space in the above classes of models and it may help discriminating among the models by then proposed. It is, therefore, important to get ready reliable calculations of this decay rate, i.e., calculations in which theoretical uncertainties are reduced as much as possible, and which are general enough to be applied to generic supersymmetric models.

The experimental situation is, at present, as follows. The ALEPH Collaboration at the CER $e^{+} e^{-}$collider LEP reports a value of the inclusive decay $\bar{B} \rightarrow X_{s} \gamma$ of [12]

$$
\mathrm{BR}\left(\bar{B} \rightarrow X_{s} \gamma\right)=(3.11 \pm 0.80 \pm 0.72) \times 10^{-4}
$$

from a sample of $b$ hadrons at the $Z$ resonance. The CLEO Collaboration at the Cornell Electron Storage Ring (CESR) has a statistically and systematically more precise result, based on $3.3 \times 10^{6} B \bar{B}$ events [13],

$$
\mathrm{BR}\left(\bar{B} \rightarrow X_{s} \gamma\right)=(3.15 \pm 0.35 \pm 0.32 \pm 0.26) \times 10^{-4},
$$

but quotes a still very large interval [13],

$$
2 \times 10^{-4}<\operatorname{BR}\left(\bar{B} \rightarrow X_{s} \gamma\right)<4.5 \times 10^{-4},
$$

as the range of acceptable values of branching ratios.

Theoretically, the rate for this decay, characterized by its large QCD contributions, practically as large as the purely 
electroweak ones [14], is known with high accuracy in the standard model (SM). It has been calculated up to the nextto-leading order (NLO) in QCD, using the formalism of effective Hamiltonians [15]. Results for LO and NLO calculations and for power corrections can be found in [16-18], [19-23], and [24], respectively. The resulting theoretical accuracy is rather astonishing: the inclusion of the NLO QCD corrections reduces the large scale dependences that are present at $\mathrm{LO}( \pm 25 \%)$ to a mere percent uncertainty, once the value of the parameters to be input in this calculation is fixed. This accuracy, however, is obtained through large and accidental numerical cancellations among different contributions to the NLO corrections and a subsequent cancellation of scale dependences $[23,25]$. The same accuracy, indeed, is not obtained for the NLO calculation of the rate $\operatorname{BR}(\bar{B}$ $\left.\rightarrow X_{s} \gamma\right)$ in simple extensions of the SM, such as models that differ from the SM by the addition of two or more doublets to the Higgs sector [23].

The calculation of $\operatorname{BR}\left(\bar{B} \rightarrow X_{s} \gamma\right)$ within supersymmetric models is still far from this level of sophistication. There are several contributions to the amplitude of this decay, usually identified by the particles exchanged in the loop. Besides the $W^{-}-t$-quark and $H^{-}-t$-quark contributions, there are also the chargino, gluino and neutralino contributions, respectively mediated by the exchange of chargino-up-squarks, gluino-down-squarks and neutralino-down-squarks. All these contributions were calculated in Ref. [26] within MSUGRA; their analytic expressions apply naturally to GMSB models also. The inclusion of QCD corrections needed for the calculation of the rate, was assumed in [26] to follow the SM pattern. No dedicated study of this decay exists for the supersymmetric models mentioned above with specific flavor symmetries. A calculation of $\operatorname{BR}\left(\bar{B} \rightarrow X_{s} \gamma\right)$ induced solely by the gluino contribution has been performed in [27,28] for a generic supersymmetric model, but no QCD corrections were included.

A NLO analysis of $\operatorname{BR}\left(\bar{B} \rightarrow X_{s} \gamma\right)$ was recently performed [29] for a specific supersymmetric case (the corresponding NLO matching conditions are also given in [30]). This is valid in a class of models where the only source of flavor violation at the electroweak scale is that of the SM, encoded in the Cabibbo-Kobayashi-Maskawa (CKM) matrix. It applies to MSUGRA and GMSB models (in which the same features are assumed/obtained at the messenger scale) only when the amount of flavor violation, generated radiatively between the supersymmetry-breaking scale and the electroweak scale, can be neglected with respect to that induced by the CKM matrix. It applies, therefore, to the case in which only the lightest stop eigenstate contributes to the chargino-mediated loop and all other squarks and gluino are heavy enough to be decoupled at the electroweak scale. It cannot be used in particular directions of parameter space of the above listed models in which quantum effects induce a gluino contribution [31] as large as the chargino or the SM contribution [11,32]. Nor can it be used as a modeldiscriminator tool, able to constrain the potentially large sources of flavor violation typical of generic supersymmetric models.
Among these, flavor-violating scalar mass terms and trilinear terms induce a flavor nondiagonal vertex gluinoquark-squark. This is generically assumed to provide the dominant contributions to quark-flavor transitions thanks to its large coupling $g_{s}$. Therefore, it is often taken as the only contribution to these transitions [33], and in particular to the $b \rightarrow s \gamma$ decay, when attempting to obtain order-of-magnitude upper bounds on flavor-violating terms in the scalar potential $[27,28]$. Once the constraints coming from experimental measurements are imposed, however, the gluino contribution is reduced to values such that the SM and the other supersymmetric contributions can no longer be neglected. Any LO and NLO calculation of the $b \rightarrow s \gamma$ rate in generic supersymmetric models should then include all possible contributions.

The gluino contribution presents some peculiar features, related to the implementation of QCD corrections, that have not been detected so far. As already mentioned, the decay $b \rightarrow s \gamma$ involves a quark-flavor violation as well as chirality violation. The first is directly related to the flavor violation in the virtual sfermions exchanged in the loop. The second can be obtained as in the SM, through a chirality flip in the external $b$ quark, and it is signaled by its mass $\bar{m}_{b}$. It can also be induced by sfermion mass terms originating from trilinear soft supersymmetry-breaking terms. These mass terms differ from fermionic mass terms by two units of $R$-charge under a $U(1)_{R}$ symmetry. The correct $R$ charge for this $b-s$ transition is then restored through the insertion of the gluino mass $m_{\tilde{g}}$ in the gluino propagator. The two different mechanisms producing chirality violation are well known. They give rise to operators of different dimensionality when generating the effective Hamiltonian used to include QCD corrections to the $b \rightarrow s \gamma$ decay. Indeed, $m_{\tilde{g}}$, the mass of one of the heavy fields exchanged in the loop, is naturally incorporated in the Wilson coefficient of the corresponding magnetic operator, which is now of dimension five $\left[e g_{s}^{2}\left(\bar{s} \sigma^{\mu \nu} P_{R} b\right) F_{\mu \nu}\right]$. On the contrary, $\bar{m}_{b}$, the running mass of one light field, with a full dynamics below the matching scale, is naturally included in the definition of a magnetic operator, which is of dimension six $\left[e g_{s}^{2} \bar{m}_{b}\left(\bar{s} \sigma^{\mu \nu} P_{R} b\right) F_{\mu \nu}\right]$.

Moreover, the presence of the strong coupling $\alpha_{s}$ in the gluino contribution immediately sparks off the question of whether this coupling should be included in the definition of the gluino-induced operators or in the corresponding Wilson coefficients. Both choices are, in principle, acceptable. It can be observed, however, as will be discussed in Sec. II, that the first option does not require a modification of the program of implementation of QCD corrections established in the SM case. In particular, the anomalous dimension matrix starts at order $\alpha_{s}$ and is used up to order $\alpha_{s}\left(\alpha_{s}^{2}\right)$ in a LO (NLO) calculation. The inclusion of the $\alpha_{s}$ coupling in the operators imposes a necessary distinction of the dimension six gluinoinduced magnetic operators $e g_{s}^{2} \bar{m}_{b}\left(\bar{s} \sigma^{\mu \nu} P_{R} b\right) F_{\mu \nu}$ from the SM magnetic operator $e / 16 \pi^{2} \bar{m}_{b}\left(\bar{s} \sigma^{\mu \nu} P_{R} b\right) F_{\mu \nu}$. As it will be seen in Sec. II, a set of new four-fermion operators, induced by gluino exchanges, is also needed.

These features single out the gluino contribution to the decay $b \rightarrow s \gamma$ as one that necessarily requires a dedicated 
study of the implementation of QCD corrections already at the LO in QCD, before including chargino and neutralino contributions and higher-order QCD corrections. In Sec. II, the list of operators induced by gluino-mediated loops is given together with the list of those needed for the SM contribution. The number of operators depends on the sources of flavor violation that are present in the particular supersymmetric model considered. In an attempt to reach the level of generality advocated above, no restriction is made on the possible sources of flavor violation in the sfermion sector. These are surveyed in Sec. III. Also shown is the direct connection between flavor-violating sources and operators generated, emphasizing the differences between the analysis in a generic supersymmetric model and the typical MSUGRAinspired analyses. The Wilson coefficients at the matching scale for the Hamiltonian generated by gluino contributions are given in Sec. IV. They are calculated using the masseigenstate formalism, the most appropriate to deal with different off-diagonal terms in the sfermion mass matrix squared, of a priori unknown size. These coefficients evolve down to the low-scale $\mu_{b}$ independently of the usual SM coefficients, since there is no mixing between SM and gluino-induced operators. The anomalous-dimension matrix governing this evolution at the $\mathrm{LO}$ in $\mathrm{QCD}$ and the resulting analytic expressions for the low-scale Wilson coefficients is given in Sec. V. In Sec. VI, an expression for the LO rate $\operatorname{BR}\left(\bar{B} \rightarrow X_{s} \gamma\right)$, due to the SM and the gluino-induced Wilson coefficients, is derived. Numerical evaluations of the branching ratio are shown in Sec. VII, when only one or at most two off-diagonal elements in the down-squark mass matrix squared are non-vanishing. As already mentioned, the decay $b \rightarrow s \gamma$ can be realistically used as a tool to select viable supersymmetric flavor models only when all contributions to $\operatorname{BR}\left(\bar{B} \rightarrow X_{s} \gamma\right)$ are included. The numerical evaluations of Sec. VII, therefore, have only the purpose of illustrating the effect of the LO QCD corrections, as well as the interplay between SM and gluino contributions to the branching ratio. Strictly speaking, they give results that are valid only in particular directions of the parameter space of generic supersymmetric models, and provide, in general, some intermediate results of an ongoing, more complete analysis.

\section{ORDERING THE QCD PERTURBATIVE EXPANSION AND THE EFFECTIVE HAMILTONIAN}

In the SM, rare $B$-meson decays are induced by loops in which $W$ bosons and up-type quarks propagate. The most important corrections are due to exchanges of light particles, gluons and light quarks, which give rise to powers of the large logarithmic factor $L=\log \left(m_{b}^{2} / m_{W}^{2}\right)$.

The decay amplitude for $b \rightarrow s \gamma$ obtains large logarithms $L$ only from loops with gluons. This implies at least one factor of $\alpha_{s}$ for each large logarithm. Since the two scales $m_{b}$ and $M_{W}$ are far apart, $L$ is a large number and these terms need to be resummed: powers of $\alpha_{s} L$ are resummed at the LO, terms of the form $\alpha_{s}\left(\alpha_{s} L\right)^{N}$ are obtained at the NLO. Thus, the corrections to the decay amplitude are classified according to

$$
\begin{gathered}
(\mathrm{LO}): G_{F}\left(\alpha_{s} L\right)^{N}, \quad(N=0,1, \ldots), \\
(\mathrm{NLO}): G_{F} \alpha_{s}\left(\alpha_{s} L\right)^{N},
\end{gathered}
$$

where $G_{F}$ is the Fermi constant.

The resummation of these corrections is usually achieved by making use of the formalism of effective Hamiltonians, combined with renormalization-group techniques. The needed effective Hamiltonian is obtained by integrating out the heavy degrees of freedom, i.e., the top-quark and the $W$ boson. It is usually expressed as

$$
\mathcal{H}_{\text {eff }}^{W}=-\frac{4 G_{F}}{\sqrt{2}} V_{t b} V_{t s}^{*} \sum_{i} C_{i}(\mu) \mathcal{O}_{i}(\mu)
$$

where $V_{t b}$ and $V_{t s}$ are elements of the Cabibbo-KobayashiMaskawa (CKM) matrix. The Wilson coefficients $C_{i}$ contain all dependence on the heavy degrees of freedom, whereas the operators $\mathcal{O}_{i}$ depend on light fields only. The operators relevant to radiative $B$ decays can be divided into two classes:

Current-current operators and gluonic penguin operators [18]:

$$
\begin{aligned}
& \mathcal{O}_{1}=\left(\bar{s} \gamma_{\mu} T^{a} P_{L} c\right)\left(\bar{c} \gamma^{\mu} T_{a} P_{L} b\right), \\
& \mathcal{O}_{2}=\left(\bar{s} \gamma_{\mu} P_{L} c\right)\left(\bar{c} \gamma^{\mu} P_{L} b\right), \\
& \mathcal{O}_{3}=\left(\bar{s} \gamma_{\mu} P_{L} b\right) \sum_{q}\left(\bar{q} \gamma^{\mu} q\right), \\
& \mathcal{O}_{4}=\left(\bar{s} \gamma_{\mu} T^{a} P_{L} b\right) \sum_{q}\left(\bar{q} \gamma^{\mu} T_{a} q\right), \\
& \mathcal{O}_{5}=\left(\bar{s} \gamma_{\mu} \gamma_{\nu} \gamma_{\rho} P_{L} b\right) \sum_{q}\left(\bar{q} \gamma^{\mu} \gamma^{\nu} \gamma^{\rho} q\right), \\
& \mathcal{O}_{6}=\left(\bar{s} \gamma_{\mu} \gamma_{\nu} \gamma_{\rho} T^{a} P_{L} b\right) \sum_{q}\left(\bar{q} \gamma^{\mu} \gamma^{\nu} \gamma^{\rho} T_{a} q\right),
\end{aligned}
$$

where $T^{a}(a=1,8)$ are $S U(3)$ color generators;

Magnetic operators, with chirality violation signaled by the presence of the $b$-quark mass:

$$
\begin{aligned}
& \mathcal{O}_{7}=\frac{e}{16 \pi^{2}} \bar{m}_{b}(\mu)\left(\bar{s} \sigma^{\mu \nu} P_{R} b\right) F_{\mu \nu}, \\
& \mathcal{O}_{8}=\frac{g_{s}}{16 \pi^{2}} \bar{m}_{b}(\mu)\left(\bar{s} \sigma^{\mu \nu} T^{a} P_{R} b\right) G_{\mu \nu}^{a},
\end{aligned}
$$

where $g_{s}$ and $e$ are the strong and electromagnetic coupling constants. Both sets of operators, those in Eqs. (5) and in (6) are of dimension six.

It is by now well known that a consistent calculation for $b \rightarrow s \gamma$ at LO (or NLO) precision requires three steps: 
(1) a matching calculation of the full standard model theory with the effective theory at the scale $\mu=\mu_{W}$ to order $\alpha_{s}^{0}\left(\right.$ or $\alpha_{s}^{1}$ ) for Wilson coefficients, where $\mu_{W}$ denotes a scale of order $M_{W}$ or $m_{t}$;

(2) a renormalization group treatment of the Wilson coefficients using the anomalous-dimension matrix to order $\alpha_{s}^{1}$ (or $\alpha_{s}^{2}$ );

(3) a calculation of the operator matrix elements at the scale $\mu=\mu_{b}$ to order $\alpha_{s}^{0}$ (or $\alpha_{s}^{1}$ ), where $\mu_{b}$ denotes a scale of order $m_{b}$.

That matters can be somewhat different is illustrated by the decay $b \rightarrow s l \bar{l}$. The effective Hamiltonian (4) contains in this case two additional operators:

$$
\begin{gathered}
\mathcal{O}_{9}=\frac{e^{2}}{16 \pi^{2}}\left(\bar{s} \gamma_{\mu} P_{L} b\right)\left(\bar{l} \gamma^{\mu} l\right), \\
\mathcal{O}_{10}=\frac{e^{2}}{16 \pi^{2}}\left(\bar{s} \gamma_{\mu} P_{L} b\right)\left(\bar{l} \gamma^{\mu} \gamma_{5} l\right) .
\end{gathered}
$$

It turns out that in this case, the operator $\mathcal{O}_{2}$ mixes into $\mathcal{O}_{9}$ at one loop: the pair $c \bar{c}$ in $\mathcal{O}_{2}$ can be closed to form a loop, and an off-shell photon producing a pair $l \bar{l}$ can be radiated from a quark line. The first large logarithm $L=\log \left(m_{b}^{2} / M_{W}^{2}\right)$ arises without the exchange of gluons. This possibility has no correspondence in the $b \rightarrow s \gamma$ case. Consequently, the decay amplitude is ordered according to $G_{F} L\left(\alpha_{s} L\right)^{N}$ at the LO in QCD and $G_{F} \alpha_{s} L\left(\alpha_{s} L\right)^{N}$ at the NLO. To achieve technically the resummation of these terms, it is convenient to redefine magnetic, chromomagnetic and lepton-pair operators $\mathcal{O}_{7}$, $\mathcal{O}_{8}, \mathcal{O}_{9}$, and $\mathcal{O}_{10}$ and the corresponding coefficients as follows [34]:

$$
\mathcal{O}_{i}^{\text {new }}=\frac{16 \pi^{2}}{g_{s}^{2}} \mathcal{O}_{i}, \quad C_{i}^{\text {new }}=\frac{g_{s}^{2}}{16 \pi^{2}} C_{i}(i=7, \ldots, 10) .
$$

This redefinition allows us to proceed according to the above three steps when calculating the amplitude of the decay $b$ $\rightarrow s l \bar{l}$ [34]. In particular, the one-loop mixing of the operator $\mathcal{O}_{2}$ with the operator $\mathcal{O}_{9}^{\text {new }}$ appears formally at $\mathcal{O}\left(\alpha_{s}\right)$.

In supersymmetric models, where the gluino-quarksquark vertex can be flavor violating, the exchange of gluino and squarks in the loop gives contribution to the decay $b$ $\rightarrow s \gamma$. Various combinations of the gluino-quark-squark vertex lead to $|\Delta(B)|=|\Delta(S)|=1$ magnetic and chromomagnetic operators (of $\mathcal{O}_{7}$-type, $\mathcal{O}_{8}$-type) with an explicit factor $\alpha_{s}$, and to four-quark operators, with a factor $\alpha_{s}^{2}$. The complete effective Hamiltonian can then be split in two terms:

$$
\mathcal{H}_{\text {eff }}=\mathcal{H}_{\text {eff }}^{W}+\mathcal{H}_{\text {eff }}^{\tilde{g}},
$$

where $\mathcal{H}_{\text {eff }}^{W}$ is the SM effective Hamiltonian in Eq. (4) and $\mathcal{H}_{\text {eff }}^{\tilde{g}}$ originates after integrating out squarks and gluinos. Note that "mixed" diagrams, which contain, besides a $W$ boson, also gluinos and squarks, give rise to $\alpha_{s}$ corrections to the Wilson coefficients in $\mathcal{H}_{\text {eff }}^{W}$ (at the matching scale). Such contributions can be omitted in a LO calculation, but they have to be taken into account at the NLO level.

As far as the gluino-induced contribution to the decay amplitude $b \rightarrow s \gamma$ is concerned, the aim is to resum the following terms:

$$
\begin{gathered}
(\mathrm{LO}): \alpha_{s}\left(\alpha_{s} L\right)^{N}, \quad(N=0,1, \ldots), \\
(\mathrm{NLO}): \alpha_{s} \alpha_{s}\left(\alpha_{s} L\right)^{N}
\end{gathered}
$$

respectively, at the leading and next-to-leading order.

While $\mathcal{H}_{e f f}^{\tilde{g}}$ is unambiguous, it is a matter of convention whether the $\alpha_{s}$ factors, peculiar to the gluino exchange, should be put into the definition of operators or into the Wilson coefficients. In analogy to the decay $b \rightarrow s l^{+} l^{-}$discussed above, it is convenient to distribute the factors of $\alpha_{s}$ between operators and Wilson coefficients in such a way that the first two of the three steps in the program for the SM calculation also apply to the gluino-induced contribution. This implies one factor of $\alpha_{s}^{1}$ in the definition of the magnetic and chromomagnetic operators and a factor $\alpha_{s}^{2}$ in the definition of the four-quark operators. With this convention, the matching calculation and the evolution down to the low scale $\mu_{b}$ of the Wilson coefficients are organized exactly in the same way as in the SM. The anomalous-dimension matrix, indeed, has the canonical expansion in $\alpha_{s}$ and starts with a term proportional to $\alpha_{s}^{1}$. The last of the three steps in the program of the SM calculation requires now an obvious modification: the calculation of the matrix elements has to be performed at order $\alpha_{s}$ and $\alpha_{s}^{2}$ at the LO and NLO precision. With this organization of QCD corrections, the SM Hamiltonian $\mathcal{H}_{\text {eff }}^{W}$ in Eq. (4) and the gluino-induced one $\mathcal{H}_{\text {eff }}^{\tilde{g}}$ undergo separate renormalization, which facilitates all considerations.

The effective Hamiltonian $\mathcal{H}_{\text {eff }}^{\tilde{g}}$, is further split into two parts:

$$
\mathcal{H}_{\text {eff }}^{\tilde{g}}=\sum_{i} C_{i, \tilde{g}}(\mu) \mathcal{O}_{i, \tilde{g}}(\mu)+\sum_{i} \sum_{q} C_{i, \tilde{g}}^{q}(\mu) \mathcal{O}_{i, \tilde{g}}^{q}(\mu),
$$

where the index $q$ runs over all light quarks $q=u, d, c, s, b$. The operators contributing to the first part are

Magnetic operators, with chirality violation coming from the $b$-quark mass:

$\mathcal{O}_{7 b, \tilde{g}}=e g_{s}^{2}(\mu) \bar{m}_{b}(\mu)\left(\bar{s} \sigma^{\mu \nu} P_{R} b\right) F_{\mu \nu}$,

$\mathcal{O}_{7 b, \tilde{g}}^{\prime}=e g_{s}^{2}(\mu) \bar{m}_{b}(\mu)\left(\bar{s} \sigma^{\mu \nu} P_{L} b\right) F_{\mu \nu}$,

$\mathcal{O}_{8 b, \tilde{g}}=g_{s}(\mu) g_{s}^{2}(\mu) \bar{m}_{b}(\mu)\left(\bar{s} \sigma^{\mu \nu} T^{a} P_{R} b\right) G_{\mu \nu}^{a}$,

$\mathcal{O}_{8 b, \tilde{g}}^{\prime}=g_{s}(\mu) g_{s}^{2}(\mu) \bar{m}_{b}(\mu)\left(\bar{s} \sigma^{\mu \nu} T^{a} P_{L} b\right) G_{\mu \nu}^{a}$,

of dimension six, as the SM operators. A contribution to the magnetic operator $\mathcal{O}_{7 b, \tilde{g}}$ is shown in Fig. 1 . In this and the 


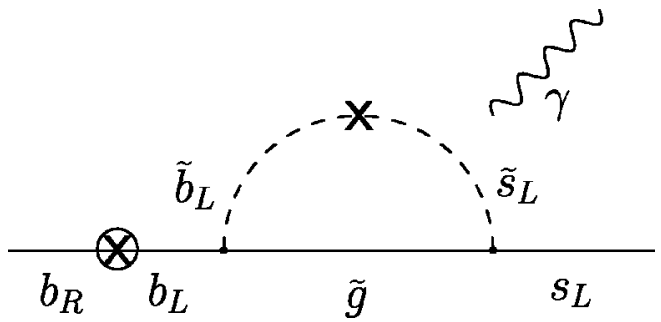

FIG. 1. Diagram mediating the $b \rightarrow s \gamma$ decay through gluino exchange and contributing to the operator $\mathcal{O}_{7 b, \tilde{g}}$. A contribution to the primed operator $\mathcal{O}_{7 b, \tilde{g}}^{\prime}$ is obtained by exchanging $L \leftrightarrow R$.

following diagrams, only the first in the series of possible insertions of chiral-flavor-violating scalar mass terms is drawn. This has the advantage of showing pictorially the correlation among supersymmetric sources of flavor violation and the generation of operators contributing to the effective Hamiltonian (10). Nevertheless, the actual calculations presented in this paper are performed using squark mass eigenstates, i.e. resumming over all possible scalar mass insertions.

Magnetic operators in which the chirality-violating parameter is the gluino mass $m_{\tilde{g}}$, included in the corresponding Wilson coefficients:

$$
\begin{aligned}
& \mathcal{O}_{7 \tilde{g}, \tilde{g}}=e g_{s}^{2}(\mu)\left(\bar{s} \sigma^{\mu \nu} P_{R} b\right) F_{\mu \nu}, \\
& \mathcal{O}_{7 \tilde{g}, \tilde{g}}^{\prime}=e g_{s}^{2}(\mu)\left(\bar{s} \sigma^{\mu \nu} P_{L} b\right) F_{\mu \nu}, \\
& \mathcal{O}_{8 \tilde{g}, \tilde{g}}=g_{s}(\mu) g_{s}^{2}(\mu)\left(\bar{s} \sigma^{\mu \nu} T^{a} P_{R} b\right) G_{\mu \nu}^{a}, \\
& \mathcal{O}_{8 \tilde{g}, \tilde{g}}^{\prime}=g_{s}(\mu) g_{s}^{2}(\mu)\left(\bar{s} \sigma^{\mu \nu} T^{a} P_{L} b\right) G_{\mu \nu}^{a} .
\end{aligned}
$$

Notice that these operators have dimension five, i.e. dimensionality lower than that of all remaining operators, of dimension six. Diagrams generating these operators are shown in Figs. 2 and 3.

Magnetic operators, with chirality violation signaled by the presence of the $c$-quark mass:

$$
\begin{aligned}
& \mathcal{O}_{7 c, \tilde{g}}=e g_{s}^{2}(\mu) \bar{m}_{c}(\mu)\left(\bar{s} \sigma^{\mu \nu} P_{R} b\right) F_{\mu \nu}, \\
& \mathcal{O}_{7 c, \tilde{g}}^{\prime}=e g_{s}^{2}(\mu) \bar{m}_{c}(\mu)\left(\bar{s} \sigma^{\mu \nu} P_{L} b\right) F_{\mu \nu}, \\
& \mathcal{O}_{8 c, \tilde{g}}=g_{s}(\mu) g_{s}^{2}(\mu) \bar{m}_{c}(\mu)\left(\bar{s} \sigma^{\mu \nu} T^{a} P_{R} b\right) G_{\mu \nu}^{a}, \\
& \mathcal{O}_{8 c, \tilde{g}}^{\prime}=g_{s}(\mu) g_{s}^{2}(\mu) \bar{m}_{c}(\mu)\left(\bar{s} \sigma^{\mu \nu} T^{a} P_{L} b\right) G_{\mu \nu}^{a} .
\end{aligned}
$$

The origin of these will become clear after discussing the second term in Eq. (10). This contains

Four-quark operators with vector Lorentz structure:

$$
\begin{aligned}
& \mathcal{O}_{11, \tilde{g}}^{q}=g_{s}^{4}(\mu)\left(\bar{s} \gamma_{\mu} P_{L} b\right)\left(\bar{q} \gamma^{\mu} P_{L} q\right), \\
& \mathcal{O}_{11, \tilde{g}}^{q^{\prime}}=g_{s}^{4}(\mu)\left(\bar{s} \gamma_{\mu} P_{R} b\right)\left(\bar{q} \gamma^{\mu} P_{R} q\right),
\end{aligned}
$$

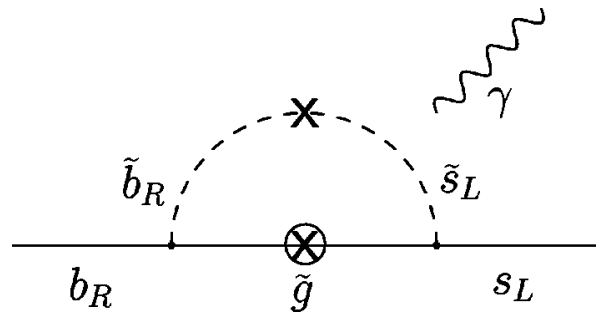

FIG. 2. Contribution to $\mathcal{O}_{7} \tilde{g}, \tilde{g}$ from the insertion of the gluino mass and of a scalar mass term simultaneously violating chirality and flavor. A contribution to $\mathcal{O}_{7 \tilde{g}, \tilde{g}}^{\prime}$ is obtained through the interchange $L \leftrightarrow R$.

$$
\begin{aligned}
& \mathcal{O}_{12, \tilde{g}}^{q}=g_{s}^{4}(\mu)\left(\bar{s}_{\alpha} \gamma_{\mu} P_{L} b_{\beta}\right)\left(\bar{q}_{\beta} \gamma^{\mu} P_{L} q_{\alpha}\right), \\
& \mathcal{O}_{12, \tilde{g}}^{q^{\prime}}=g_{s}^{4}(\mu)\left(\bar{s}_{\alpha} \gamma_{\mu} P_{R} b_{\beta}\right)\left(\bar{q}_{\beta} \gamma^{\mu} P_{R} q_{\alpha}\right), \\
& \mathcal{O}_{13, \tilde{g}}^{q}=g_{s}^{4}(\mu)\left(\bar{s} \gamma_{\mu} P_{L} b\right)\left(\bar{q} \gamma^{\mu} P_{R} q\right), \\
& \mathcal{O}_{13, \tilde{g}}^{q^{\prime}}=g_{s}^{4}(\mu)\left(\bar{s} \gamma_{\mu} P_{R} b\right)\left(\bar{q} \gamma^{\mu} P_{L} q\right), \\
& \mathcal{O}_{14, \tilde{g}}^{q}=g_{s}^{4}(\mu)\left(\bar{s}_{\alpha} \gamma_{\mu} P_{L} b_{\beta}\right)\left(\bar{q}_{\beta} \gamma^{\mu} P_{R} q_{\alpha}\right), \\
& \mathcal{O}_{14, \tilde{g}}^{q^{\prime}}=g_{s}^{4}(\mu)\left(\bar{s}_{\alpha} \gamma_{\mu} P_{R} b_{\beta}\right)\left(\bar{q}_{\beta} \gamma^{\mu} P_{L} q_{\alpha}\right),
\end{aligned}
$$

where color indices are omitted for color-singlet currents. They arise from box diagrams through the exchange of two gluinos and from penguin diagrams through the exchange of a gluino and a gluon. A typical penguin diagram is shown in Fig. 4. According to their Lorentz structure, these operators will be called hereafter vector four-quark operators.

Four-quark operators with scalar and tensor Lorentz structure:

$$
\begin{aligned}
& \mathcal{O}_{15, \tilde{g}}^{q}=g_{s}^{4}(\mu)\left(\bar{s} P_{R} b\right)\left(\bar{q} P_{R} q\right), \\
& \mathcal{O}_{15, \tilde{g}}^{q^{\prime}}=g_{s}^{4}(\mu)\left(\bar{s} P_{L} b\right)\left(\bar{q} P_{L} q\right), \\
& \mathcal{O}_{16, \tilde{g}}^{q}=g_{s}^{4}(\mu)\left(\bar{s}_{\alpha} P_{R} b_{\beta}\right)\left(\bar{q}_{\beta} P_{R} q_{\alpha}\right), \\
& \mathcal{O}_{16, \tilde{g}}^{q^{\prime}}=g_{s}^{4}(\mu)\left(\bar{s}_{\alpha} P_{L} b_{\beta}\right)\left(\bar{q}_{\beta} P_{L} q_{\alpha}\right), \\
& \mathcal{O}_{17, \tilde{g}}^{q}=g_{s}^{4}(\mu)\left(\bar{s} P_{R} b\right)\left(\bar{q} P_{L} q\right), \\
& \mathcal{O}_{17, \tilde{g}}^{q^{\prime}}=g_{s}^{4}(\mu)\left(\bar{s} P_{L} b\right)\left(\bar{q} P_{R} q\right), \\
& \mathcal{O}_{18, \tilde{g}}^{q}=g_{s}^{4}(\mu)\left(\bar{s}_{\alpha} P_{R} b_{\beta}\right)\left(\bar{q}_{\beta} P_{L} q_{\alpha}\right), \\
& \mathcal{O}_{18, \tilde{g}}^{q^{\prime}}=g_{s}^{4}(\mu)\left(\bar{s}_{\alpha} P_{L} b_{\beta}\right)\left(\bar{q}_{\beta} P_{R} q_{\alpha}\right), \\
& \mathcal{O}_{19, \tilde{g}}^{q}=g_{s}^{4}(\mu)\left(\bar{s} \sigma_{\mu \nu} P_{R} b\right)\left(\bar{q} \sigma^{\mu \nu} P_{R} q\right), \\
& \mathcal{O}_{19, \tilde{g}}^{q^{\prime}}=g_{s}^{4}(\mu)\left(\bar{s} \sigma_{\mu \nu} P_{L} b\right)\left(\bar{q} \sigma^{\mu \nu} P_{L} q\right),
\end{aligned}
$$



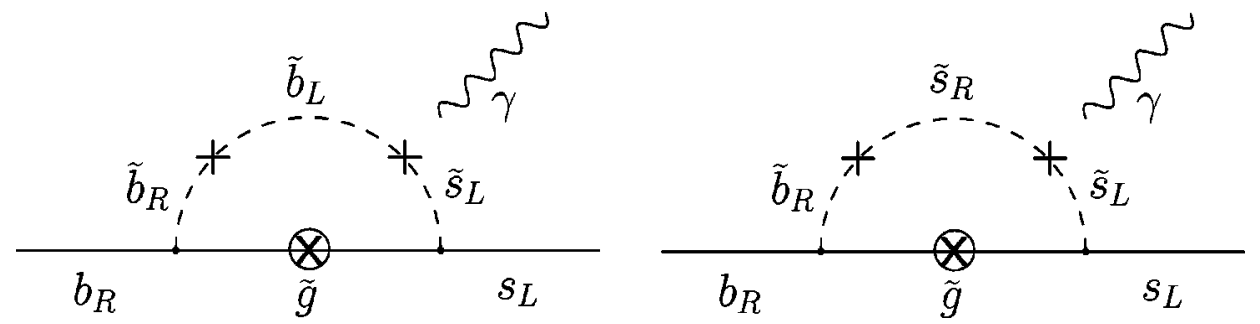

FIG. 3. Contributions to $\mathcal{O}_{7} \tilde{g}, \tilde{g}$ from the insertion of the gluino mass and distinct chirality- and flavor-violating scalar mass terms. In the approximation $m_{s}=0$, the second diagram requires trilinear terms not linked to Yukawa couplings. The analogous contributions to $\mathcal{O}_{7 \tilde{g}, \tilde{g}}^{\prime}$ are obtained through the interchange $L \leftrightarrow R$.

$$
\begin{aligned}
& \mathcal{O}_{20, \tilde{g}}^{q}= g_{s}^{4}(\mu)\left(\bar{s}_{\alpha} \sigma_{\mu \nu} P_{R} b_{\beta}\right)\left(\bar{q}_{\beta} \sigma^{\mu \nu} P_{R} q_{\alpha}\right), \\
& \mathcal{O}_{20, \tilde{g}}^{q^{\prime}}=g_{s}^{4}(\mu)\left(\bar{s}_{\alpha} \sigma_{\mu \nu} P_{L} b_{\beta}\right)\left(\bar{q}_{\beta} \sigma^{\mu \nu} P_{L} q_{\alpha}\right),
\end{aligned}
$$

which are induced by box diagrams only and through the exchange of two gluinos. Examples of box diagrams are sketched in Figs. 5. In the following, the operators (15) will be called scalar-tensor four-quark operators. Notice that, for different $q$ 's, $\mathcal{O}_{11, \tilde{g}}^{q}-\mathcal{O}_{20, \tilde{g}}^{q}$ are in general distinct sets of operators.

The four-quark operators in Eqs. (14) and (15) are formally of higher order in the strong coupling than the magnetic and chromomagnetic operators (11)-(13). As it will be explicitly shown in Sec. IV, the scalar-tensor operators $\mathcal{O}_{15, \tilde{g}}^{q}-\mathcal{O}_{20 \tilde{g}}^{q}$ mix at one loop into the magnetic and chromomagnetic operators. Given this fact, the necessity of including $\mathcal{O}_{7 c, \tilde{g}}$ and $\mathcal{O}_{8 c, \tilde{g}}$ in the operator basis becomes clear immediately: some of the operators $\mathcal{O}_{15, \tilde{g}}^{q}, \ldots, \mathcal{O}_{20, \tilde{g}}^{q}$ with $q$ $=c$ mix into $\mathcal{O}_{7 c, \tilde{g}}$ and $\mathcal{O}_{8 c, \tilde{g}}$. Such mixing terms can be calculated by considering the one-loop matrix elements $\left\langle s \gamma\left|\mathcal{O}_{i, \tilde{g}}^{c}\right| b\right\rangle$ and $\left\langle s g\left|\mathcal{O}_{i, \tilde{g}}^{c}\right| b\right\rangle(i=15, \ldots, 20)$, respectively. In principle, also operators like $\mathcal{O}_{7 u, \tilde{g}}, \mathcal{O}_{7 d, \tilde{g}}$ and $\mathcal{O}_{7 s, \tilde{g}}$ are induced in an analogous way. These operators, however, are weighted by $m_{u}, m_{d}$ and $m_{s}$ and are vanishing in the approximation used here: $m_{u}=m_{d}=m_{s}=0$.

Due to these mixing effects, the scalar-tensor operators have to be included in a LO calculation for the decay amplitude. The remaining four-quark operators with vector structure $\mathcal{O}_{11, \tilde{g}}^{q}-\mathcal{O}_{14, \tilde{g}}^{q}$ (and the corresponding primed operators) do not mix at one loop neither into the magnetic and chromomagnetic operators nor into the four-quark operators

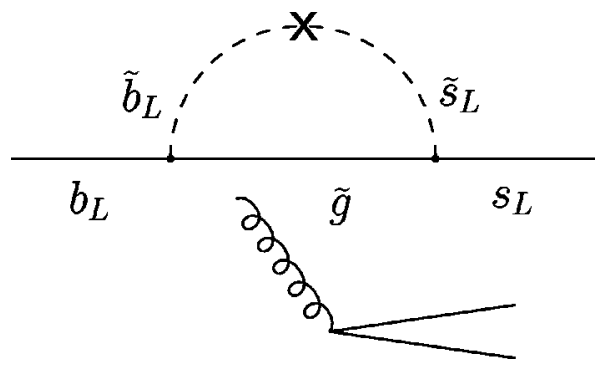

FIG. 4. Penguin diagram contributing to the operators (14).
$\mathcal{O}_{15, \tilde{g}}^{q}-\mathcal{O}_{20, \tilde{g}}^{q}$. Therefore, these vector four-quark operators become relevant only at the NLO precision.

We end this section with a comment on the definition of the strong coupling constant used in the various steps of the calculation. In the full theory, which consists here of the SM and gluino-down-squark sectors of a supersymmetric model, all particles contribute to the running of this coupling, indicated by the symbol $\hat{g}_{s}(\mu)$. In order to perform the matching with the effective theory, where only the five light quarks survive, all the heavy particles have to be decoupled. The strong coupling constant in this regime, indicated by $g_{s}(\mu)$, differs from $\hat{g}_{s}(\mu)$ by logarithmic terms signaling the decoupling of the heavy particles:

$$
\hat{g}_{s}(\mu)=g_{s}(\mu)\left[1+g_{s}^{2}(\mu)\left(\text { decoupling } \log ^{\prime} \mathrm{s}\right)\right] .
$$

At NLO precision, these decoupling terms have to be taken into account explicitly. At LO precision, however, $\hat{g}_{s}(\mu)$ and $g_{s}(\mu)$ can be identified and $g_{s}(\mu)$ is here always understood to be the modified minimal subtraction scheme ( $\overline{\mathrm{MS}})$ strong coupling at the renormalization scale $\mu$, running with five flavors.

\section{SOURCES OF FLAVOR VIOLATION}

Supersymmetric models contain all sources of flavor violation present in a two Higgs doublet model of type II, i.e. the vertices with a charged boson: $\bar{u}_{L i}-d_{L j}-W^{+}$and $\bar{u}_{L i}{ }^{-}$ $d_{R j}-H^{+}, \bar{u}_{R i}-d_{L j}-H^{+}(i, j=1,2,3)$. Once the electroweak symmetry is broken, a rotation in flavor space [35]

$$
D^{o}=V_{d} D, \quad U^{o}=V_{u} U, \quad D^{c o}=U_{d}^{*} D^{c}, \quad U^{c o}=U_{u}^{*} U^{c},
$$

of all matter superfields in the superpotential

$$
W=-D_{i}^{c o}\left(h_{d}\right)_{i j} Q_{j}^{o} H_{d}+U_{i}^{c o}\left(h_{u}\right)_{i j} Q_{j}^{o} H_{u}-\mu H_{d} H_{u}
$$

brings fermions from the current eigenstate basis $\left\{d_{L}^{o}, u_{L}^{o}, d_{R}^{o}, u_{R}^{o}\right\} \quad$ to their mass eigenstate basis $\left\{d_{L}, u_{L}, d_{R}, u_{R}\right\}$ :

$$
d_{L}^{o}=V_{d} d_{L}, \quad u_{L}^{o}=V_{u} u_{L}, \quad d_{R}^{o}=U_{d} d_{R}, \quad u_{R}^{o}=U_{u} u_{R},
$$




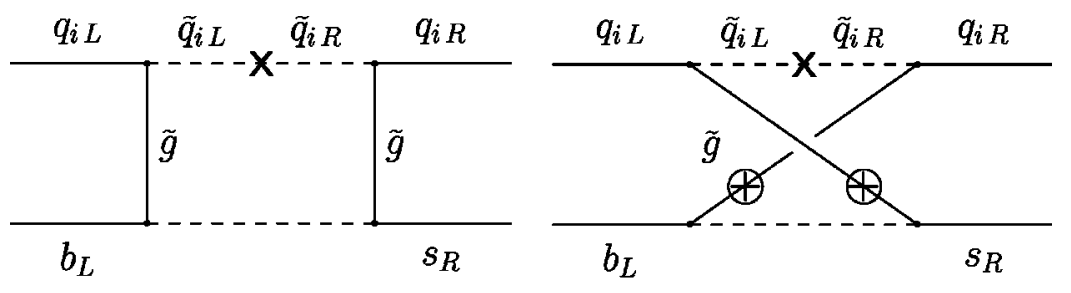

FIG. 5. Diagrams contributing to the operators (15). In the two upper diagrams, the quark (squark) $q(\tilde{q})$ can be of up- or down-type and the flavor violation on the lower squark line, not explicitly indicated, can be realized through a direct flavor-chiral transition (see Fig. 2) or through

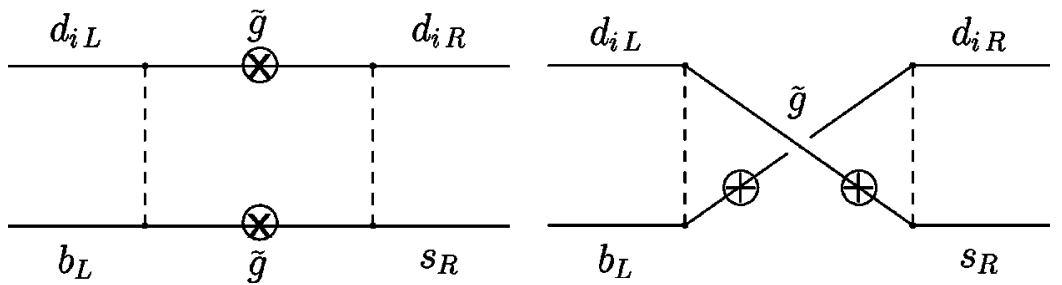
distinct chirality and flavor transitions (see Fig. $3)$. In the lower diagrams, the down-type quark $d_{i}$ is a $b$-or an $s$-quark if a single flavor violation is allowed in the squark lines.

and the scalar superpartners to the basis $\left\{\widetilde{D}, \widetilde{U}, \widetilde{D}^{c}, \widetilde{U}^{c}\right\}$. Through this rotation, the Yukawa matrices $h_{d}$ and $h_{u}$ are reduced to their diagonal form $\hat{h}_{d}$ and $\hat{h}_{u}$ :

$$
\left(\hat{h}_{d}\right)_{i i}=\left(U_{d}^{\dagger} h_{d} V_{d}\right)_{i i}=\frac{m_{d i}}{v_{d}}, \quad\left(\hat{h}_{u}\right)_{i i}=\left(U_{u}^{\dagger} h_{u} V_{u}\right)_{i i}=\frac{m_{u i}}{v_{u}} .
$$

Tree-level mixing terms among quarks of different generations are due to the misalignment of $V_{d}$ and $V_{u}$; all the above vertices $\bar{u}_{L i}-d_{L j}-W^{+}$and $\bar{u}_{L i}-d_{R j}-H^{+}, \bar{u}_{R i}-d_{L j}-$ $H^{+}(i, j=1,2,3)$ are weighted by the elements of the CKM matrix $V=V_{u}^{\dagger} V_{d}$. The supersymmetric counterpart of these vertices, $\bar{u}_{L i}-\widetilde{D}_{j}-\widetilde{W}^{+}, \bar{u}_{L i}-\widetilde{D}_{j}^{c} *-\widetilde{H}^{+}, \bar{u}_{R i}-\widetilde{D}_{j}-\widetilde{H}^{-}$, are also proportional to $V_{i j}$ in the limit of unbroken supersymmetry.

To illustrate the sources of flavor violation that may be present in supersymmetric models in addition to those encoded in the CKM matrix, it is instructive to consider in detail the contributions to the squared-mass matrix of a squark of flavor $f$. The relation between off-diagonal terms in this squared-mass matrix and the type of operators inducing the decay $b \rightarrow s \gamma$, will then become clear. Since present collider limits give indications that the squark masses are larger than those of the corresponding quarks, the largest entries in the squark mass matrices squared must come from the soft potential, directly linked to the mechanism of supersymmetry breaking. When restricted to the terms relevant to squark masses and quark-flavor transitions, the soft potential can be expressed in terms of the current eigenstates scalar fields as

$$
\begin{aligned}
V_{\text {soft }} \supset \widetilde{Q}_{i}^{o *} & m_{\widetilde{Q} i j}^{2} \widetilde{Q}_{j}^{o}+\widetilde{D}_{i}^{c o *} m_{\widetilde{D} i j}^{2} \widetilde{D}_{j}^{c o}+\widetilde{U}_{i}^{c o} * m_{\widetilde{U} i j}^{2} \widetilde{U}_{j}^{c o} \\
& +\left(-\frac{1}{2} M_{3} \lambda_{3} \lambda_{3}+A_{d, i j} H_{d} \widetilde{Q}_{i}^{o} \widetilde{D}_{j}^{c o}\right. \\
& \left.+A_{u, i j} H_{u} \widetilde{Q}_{i}^{o} \widetilde{U}_{j}^{c o}+\text { H.c. }\right) .
\end{aligned}
$$

In Eq. (21), $m_{\widetilde{Q}}^{2}, m_{\widetilde{D}}^{2}$, and $m_{\widetilde{U}}^{2}$ are Hermitian matrices. The gluino $\tilde{g}$, a four-component Majorana spinor, is expressed in terms of the Weyl spinor $\lambda_{3}$ and has mass $m_{\tilde{g}}=M_{3}$. Notice that, for the trilinear terms $A_{d, i j} H_{d} \widetilde{Q}_{i}^{o} \widetilde{D}_{j}^{c o}$, no proportionality to the Yukawa couplings is assumed. These trilinear scalar terms are left completely general and may also represent non-holomorphic ones, of the type $A_{d, i j}^{\prime} H_{u}^{*} \widetilde{Q}_{i}^{o} \widetilde{D}_{j}^{c o}$ discussed in [36].

Thus, in the interaction basis $\left(\widetilde{Q}_{1}^{o}, \widetilde{Q}_{2}^{o}, \widetilde{Q}_{3}^{o}, \widetilde{Q}_{1}^{c o *}, \widetilde{Q}_{2}^{c o *}, \widetilde{Q}_{3}^{c o *}\right)$, often denoted also as $\left(\tilde{q}_{L 1}^{o}, \tilde{q}_{L 2}^{o}, \tilde{q}_{L 3}^{o}, \tilde{q}_{R 1}^{o}, \tilde{q}_{R 2}^{o}, \tilde{q}_{R 3}^{o}\right)$, the squared-mass matrix for a squark of flavor $f$ has the form

$$
\mathcal{M}_{f}^{2} \equiv\left(\begin{array}{cc}
m_{f, L L}^{2}+F_{f L L}+D_{f L L} & \left(m_{f, L R}^{2}\right)+F_{f L R} \\
\left(m_{f, L R}^{2}\right)^{\dagger}+F_{f R L} & m_{f, R R}^{2}+F_{f R R}+D_{f R R}
\end{array}\right) .
$$

The term $m_{f, L L}^{2}$ is $m_{\widetilde{Q}}^{2}$, for both, up- and down-type squarks; $m_{f, R R}^{2}$ is $m_{\widetilde{D}}^{2}$ for a down-type squark and $m_{\widetilde{U}}^{2}$ for an up-type squark. The off-diagonal $3 \times 3$ block matrix $m_{f, L R}^{2}$ is $A_{d}^{*} v_{d}$ for a down squark, $A_{u}^{*} v_{u}$ for an up-type one. (The two vacuum expectation values are chosen to be real.) It should be stressed that, differently from $m_{f, L L}^{2}$ and $m_{f, R R}^{2}$, the off-diagonal $3 \times 3$ matrix $m_{f, L R}^{2}$ is not Hermitian. In other words, it is $A_{d, i j} \neq A_{d, j i}^{*}$ as well as $A_{u, i j} \neq A_{u, j i}^{*}$.

The $D$-term contributions $D_{f L L}$ and $D_{f R R}$ to the squaredmass matrix (22),

$$
D_{f L L, R R}=\cos 2 \beta M_{Z}^{2}\left(T_{f}^{3}-Q_{f} \sin ^{2} \theta_{W}\right) \mathbb{1}_{3},
$$

are diagonal in flavor space.

The explicit form for the $F$-term contributions can be obtained from scalar quartic couplings arising from the superpotential (18):

$$
\begin{aligned}
V_{F} \supset & v_{d}^{2} \widetilde{D}_{i}^{o *}\left(h_{d}^{\dagger} h_{d}\right)_{i j} \widetilde{D}_{j}^{o}+v_{d}^{2} \widetilde{D}_{i}^{c o}\left(h_{d} h_{d}^{\dagger}\right)_{i j} \widetilde{D}_{j}^{c o *} \\
& -\left(\mu v_{u} \widetilde{D}_{i}^{o} * h_{d, i j}^{\dagger} \widetilde{D}_{j}^{c o *}+\text { H.c. }\right)+v_{u}^{2} \widetilde{U}_{i}^{o *}\left(h_{u}^{\dagger} h_{u}\right)_{i j} \widetilde{U}_{j}^{o} \\
& +v_{u}^{2} \widetilde{U}_{i}^{c o}\left(h_{u} h_{u}^{\dagger}\right)_{i j} \widetilde{U}_{j}^{c o *}-\left(\mu v_{d} \widetilde{U}_{i}^{o *} h_{u, i j}^{\dagger} \widetilde{U}_{j}^{c o *}+\text { H.c. }\right) .
\end{aligned}
$$


The rotation (17) reduces $F_{f L L}$ and $F_{f R R}$ to their diagonal form

$$
m_{d i}^{2} \widetilde{D}_{i}^{*} \widetilde{D}_{i}, \quad m_{u i}^{2} \widetilde{U}_{i}^{*} \widetilde{U}_{i}, \quad m_{d i}^{2} \widetilde{D}_{i}^{c *} \widetilde{D}_{i}^{c}, \quad m_{u i}^{2} \widetilde{U}_{i}^{c *} \widetilde{U}_{i}^{c},
$$

as well as $F_{f L R}\left(F_{f R L}=F_{f L R}^{\dagger}\right)$ to

$$
-\mu\left(m_{d, i} \tan \beta\right) \widetilde{D}_{i}^{*} \widetilde{D}_{i}^{c *}, \quad-\mu\left(m_{u, i} \cot \beta\right) \widetilde{U}_{i}^{*} \widetilde{U}_{i}^{c *} .
$$

Therefore, once up- and down-quarks are brought to their mass eigenstate basis through the rotation (17), the only sources of flavor violation in the squark sector arise from the off-diagonal terms in the soft mass matrices $m_{f, L L}^{2}, m_{f, R R}^{2}$, and $m_{f, L R}^{2} .{ }^{1}$ Their origin, as their magnitude, is a modeldependent matter based on the interplay between the dynamics of flavor and that dictating the breaking of supersymmetry. In general, however, they give rise to large flavor-quark transitions at the loop level, through large couplings of gluinos to quarks and squarks belonging to different generations.

One very drastic approach to this supersymmetric flavor problem is that of MSUGRA. In this model (or class of models) the soft potential (21) is characterized at some high scale, typically a grand unification scale, by the universality of the scalar masses:

$$
m_{\tilde{Q}_{i j}}=m_{\tilde{U} i j}=m_{\tilde{D} i j}=\tilde{m} \delta_{i j} ;
$$

and the proportionality of the trilinear terms to the Yukawa couplings, through a universal parameter $A$ :

$$
A_{d, i j}=A h_{d, i j} ; A_{u, i j}=A h_{u, i j} .
$$

At this high scale, the only source of flavor violation is contained in the superpotential, indicating that the breaking of supersymmetry occurs at a scale where the dynamics of flavor has already taken place.

An elegant solution to the flavor problem is obtained in GMSB models, in which the signal of supersymmetry breaking is transmitted to the visible sector of fields $\widetilde{Q}^{o}, \widetilde{U}^{o}, \widetilde{D}^{o}$, $H_{1}, H_{2}$, etc., by flavor-blind gauge interactions. In these models, at the scale of supersymmetry breaking, all matrices in Eq. (25) are diagonal, although different, and the common value of $A$ in Eq. (26) is set to zero.

In both MSUGRA and GMSB models, sources of flavor violation in the scalar sector are generated radiatively at the electroweak scale through the scalar quartic couplings proportional to Yukawa matrices. A simple inspection shows that intergenerational mixing terms due to only one type of Yukawa matrix, get eliminated by the rotation (17): no offdiagonal terms are therefore possible in $m_{f, R R}^{2}$ in these models. On the contrary, flavor-violating terms are not rotated away in the $m_{f, L L}^{2}$ sector in which radiative contributions arise from quartic scalar couplings proportional to both ma-

\footnotetext{
${ }^{1}$ No new symbols are introduced to indicate the unknown matrices $m_{f, L L}^{2}, m_{f, R R}^{2}$, and $m_{f, L R}^{2}$ after the rotation (17). Notice, however, that $m_{u, L L}^{2}$ and $m_{d, L L}^{2}$, equal before this rotation, are now related as $m_{u, L L}^{2}=V m_{d, L L}^{2} V^{\dagger}$.
}

trices $h_{d}$ and $h_{u}$. Being loop-induced, this source of flavor violation is, in general, small [26], but it becomes nonnegligible for large values of $\tan \beta$ [32]. By this reasoning it becomes clear that, while a contribution to the operator $\mathcal{O}_{7 b, \tilde{g}}$ can arise from an off-diagonal term mixing the second- and third-generation left squarks $\left(m_{d, L L}^{2}\right)_{23}$, as shown in Fig. 1, no contribution to $\mathcal{O}_{7 b, \tilde{g}}^{\prime}$ is possible in the MSUGRA and GMSB models. The same holds for all other primed operators. These operators may nevertheless acquire nonvanishing contributions in more general models, in which, for example, there exists an off-diagonal term $\left(m_{d, R R}^{2}\right)_{23}$.

Also vanishing, in MSUGRA and GMSB models, is the contribution to the operator $\mathcal{O}_{7 \tilde{g}, \tilde{g}}$ coming from a left-right mixing element $\left(m_{d, L R}^{2}\right)_{23}$. A contribution to this operator can, however, be induced, even in these models, by intergenerational mixing terms in $m_{d_{i} L L}^{2},\left(m_{d, L L}^{2}\right)_{23}$, and the flavordiagonal left-right term $\left(m_{d, L R}^{2}\right)_{33}$. In the mass-insertion formalism, often used for the calculation of supersymmetric contributions to FCNC processes [38], the first nonvanishing contribution to $\mathcal{O}_{7 \tilde{g}, \tilde{g}}$ is then generated by the double insertion shown in the first diagram of Fig. 3. It will be shown later that, in generic supersymmetric models, this contribution to $\mathcal{O}_{7 \tilde{g}, \tilde{g}}$ turns out to give the strongest constraint on $\left(m_{d, L L}^{2}\right)_{23}$, when reasonable values of $\left(m_{d, L R}^{2}\right)_{33}$ are chosen.

As advocated in the Introduction, the aim of this paper is to provide a calculation as general as possible of the gluino contribution to the decay $b \rightarrow s \gamma$, i.e. a calculation that applies to supersymmetric models with the most general soft terms. The QCD-corrected branching ratio for this decay can then be used to constrain the size of the off-diagonal elements of the mass matrices $m_{d, L L}^{2}, m_{d, R R}^{2}$, and $m_{d, L R}^{2}$. Since different operators contribute to this decay, with different numerical impact on its rate, some of these flavorviolating terms may turn out to be poorly constrained. Thus, given the generality of such a calculation, it is convenient to rely on the mass eigenstate formalism, which remains valid even when the intergenerational mixing elements are large. The procedure used follows closely Refs. $[37,26]$. The diagonalization of the two $6 \times 6$ squark mass matrices squared $\mathcal{M}_{d}^{2}$ and $\mathcal{M}_{u}^{2}$ yields the eigenvalues $m_{\tilde{d}_{k}}^{2}$ and $m_{\tilde{u}_{k}}^{2}(k$ $=1, \ldots, 6)$. The corresponding mass eigenstates, $\tilde{u}_{k}$ and $\widetilde{d}_{k}$ $(k=1, \ldots, 6)$ are related to the fields $\tilde{u}_{L j}, \tilde{u}_{R j}$ and $\widetilde{d}_{L j}, \widetilde{d}_{R j}$ $(j=1, \ldots, 3)$ as

$$
\tilde{u}_{L, R}=\Gamma_{U L, R}^{\dagger} \tilde{u}, \tilde{d}_{L, R}=\Gamma_{D L, R}^{\dagger} \tilde{d},
$$

where the four matrices $\Gamma_{U L, R}$ and $\Gamma_{D L, R}$ are $6 \times 3$ mixing matrices. The gluino-quark-squark vertices are explicitly given in Ref. [26].

\section{WILSON COEFFICIENTS AT THE ELECTROWEAK SCALE}

At the matching scale $\mu_{W}$, the non-vanishing Wilson coefficients for the SM operators in Eqs. (5) and (6) are, at leading order in $\alpha_{s}$ 


$$
\begin{aligned}
C_{2}\left(\mu_{W}\right)= & 1, \\
C_{7}\left(\mu_{W}\right)= & \frac{x_{t w}}{24\left(x_{t w}-1\right)^{4}}\left[-8 x_{t w}^{3}+3 x_{t w}^{2}+12 x_{t w}-7\right. \\
& \left.+\left(18 x_{t w}^{2}-12 x_{t w}\right) \ln x_{t w}\right] \\
C_{8}\left(\mu_{W}\right)= & \frac{x_{t w}}{8\left(x_{t w}-1\right)^{4}}\left(-x_{t w}^{3}+6 x_{t w}^{2}-3 x_{t w}-2\right. \\
& \left.-6 x_{t w} \ln x_{t w}\right),
\end{aligned}
$$

with $x_{t w} \equiv m_{t}^{2} / M_{W}^{2}$.

Among the coefficients arising from the virtual exchange of a gluino at the matching scale, the non-vanishing ones are ${ }^{2}$

$$
\begin{aligned}
& C_{7 b, \tilde{g}}\left(\mu_{W}\right)=-\frac{e_{d}}{16 \pi^{2}} C(R) \sum_{k=1}^{6} \frac{1}{m_{\tilde{d}_{k}}^{2}}\left(\Gamma_{D L}^{k b} \Gamma_{D L}^{* k s}\right) F_{2}\left(x_{g d_{k}}\right), \\
& C_{7 \tilde{g}, \tilde{g}}\left(\mu_{W}\right)=m_{\tilde{g}} \frac{e_{d}}{16 \pi^{2}} C(R) \sum_{k=1}^{6} \frac{1}{m_{\tilde{d}_{k}}^{2}}\left(\Gamma_{D R}^{k b} \Gamma_{D L}^{* k s}\right) F_{4}\left(x_{g d_{k}}\right),
\end{aligned}
$$

in the case of magnetic operators and

$$
\begin{aligned}
C_{8 b, \tilde{g}}\left(\mu_{W}\right)= & -\frac{1}{16 \pi^{2}} \sum_{k=1}^{6} \frac{1}{m_{\tilde{d}_{k}}^{2}}\left(\Gamma_{D L}^{k b} \Gamma_{D L}^{* k s}\right)\{[C(R) \\
& \left.\left.-\frac{1}{2} C(G)\right] F_{2}\left(x_{g d_{k}}\right)-\frac{1}{2} C(G) F_{1}\left(x_{g d_{k}}\right)\right\}, \\
C_{8 \tilde{g}, \tilde{g}}\left(\mu_{W}\right)= & m_{\tilde{g}} \frac{1}{16 \pi^{2}} \sum_{k=1}^{6} \frac{1}{m_{\tilde{d}_{k}}^{2}}\left(\Gamma_{D R}^{k b} \Gamma_{D L}^{* k s}\right)\{[C(R) \\
& \left.\left.-\frac{1}{2} C(G)\right] F_{4}\left(x_{g d_{k}}\right)-\frac{1}{2} C(G) F_{3}\left(x_{g d_{k}}\right)\right\},
\end{aligned}
$$

in the case of chromomagnetic operators. The coefficients $C_{7, \tilde{g}, \tilde{g}}\left(\mu_{W}\right)$ and $C_{8} \tilde{g}, \tilde{g}\left(\mu_{W}\right)$ are of higher dimensionality to compensate the lower dimensionality of the corresponding operators. The ratios $x_{g d_{k}}$ are now defined as $x_{g d_{k}} \equiv m_{g}^{2} / m_{\tilde{d}_{k}}^{2}$; the Casimir factors $C(R)$ and $C(G)$ are, respectively, $C(R)=4 / 3$ and $C(G)=3$; and the functions $F_{i}(x), i$ $=1, \ldots, 4$, are given in Appendix A. The Wilson coefficients of the corresponding primed operators are obtained through the interchange $\Gamma_{D R}^{i j} \leftrightarrow \Gamma_{D L}^{i j}$ in Eqs. (29) and (30). The coefficients of the magnetic and chromomagnetic operators, proportional to the $c$-quark mass, vanish at the matching scale at lowest order in $\alpha_{s}$.

\footnotetext{
${ }^{2}$ The linear combination $\quad C_{7 b, \tilde{g}}\left(\mu_{W}\right) \mathcal{O}_{7 b, \tilde{g}}\left(\mu_{W}\right)$ $+C_{7 \tilde{g}, \tilde{g}}\left(\mu_{W}\right) \mathcal{O}_{7 \tilde{g}, \tilde{g}}\left(\mu_{W}\right) \quad$ coincides with the expression $\delta C_{7}\left(\mu_{W}\right) \mathcal{O}_{7}\left(\mu_{W}\right)$ given in the literature (see e.g. [26,39]), where $\mathcal{O}_{7}$ is the standard model operator.
}

Compared to the SM, there is a larger number of magnetic and chromomagnetic operators with different chirality and dimensionality. The different chiralities are due to the fact that the gluino couples both to left- and right-handed quarks and the associated squarks. In contrast, the $W$ has only lefthanded couplings and therefore right-handed fields only arise if their masses are not neglected; usually only (chromo)magnetic operators with right-handed $b$ quarks are included. Similarly, the occurence of (chromo)magnetic operators with differing dimensions can also be understood from the chirality structure of the gluino couplings. Some of the new operators differ from the SM (chromo)magnetic operators only by an additional factor $g_{s}^{2}$. These were introduced as additional operators for practical reasons.

Penguin diagrams mediated by the virtual exchange of a gluino and a gluon, yield non-vanishing coefficients only for the operators $\mathcal{O}_{11, \tilde{g}}^{q}-\mathcal{O}_{14, \tilde{g}}^{q}$ :

$$
\begin{aligned}
C_{11, \tilde{g}}^{q}\left(\mu_{W}\right)= & \frac{1}{16 \pi^{2}} \frac{1}{3} \sum_{k=1}^{6} \frac{1}{m_{\tilde{d}_{k}}^{2}}\left(\Gamma_{D L}^{k b} \Gamma_{D L}^{* k s}\right)\{[C(R) \\
& \left.\left.-\frac{1}{2} C(G)\right] F_{6}\left(x_{g d_{k}}\right)+\frac{1}{2} C(G) F_{5}\left(x_{g d_{k}}\right)\right\}, \\
C_{12, \tilde{g}}^{q}\left(\mu_{W}\right)= & -\frac{1}{16 \pi^{2}} \sum_{k=1}^{6} \frac{1}{m_{\tilde{d}_{k}}^{2}}\left(\Gamma_{D L}^{k b} \Gamma_{D L}^{* k s}\right)\{[C(R) \\
& \left.\left.-\frac{1}{2} C(G)\right] F_{6}\left(x_{g d_{k}}\right)+\frac{1}{2} C(G) F_{5}\left(x_{g d_{k}}\right)\right\}, \\
C_{13, \tilde{g}}^{q}\left(\mu_{W}\right)= & C_{11, \tilde{g}}^{q}\left(\mu_{W}\right), \\
C_{14, \tilde{g}}^{q}\left(\mu_{W}\right)= & C_{12, \tilde{g}}^{q}\left(\mu_{W}\right),
\end{aligned}
$$

as well as coefficients for the corresponding primed operators, $\mathcal{O}_{11, \tilde{g}}^{q^{\prime}}-\mathcal{O}_{14, \tilde{g}}^{q^{\prime}}$, which can be obtained from those in Eq. (31) by interchanging $\Gamma_{D R}^{i j} \leftrightarrow \Gamma_{D L}^{i j}$. These coefficients are actually independent of the quark label $q$.

Box diagrams, ${ }^{3}$ with exchange of two virtual gluinos, yield the following contributions to the coefficients $C_{11, \tilde{g}^{-}}^{q}$ $C_{14, \tilde{g}}^{q}$ :

$$
\begin{aligned}
C_{11, \tilde{g}}^{q}\left(\mu_{W}\right)= & \frac{1}{16 \pi^{2}} \frac{1}{m_{\tilde{g}}^{2}} \sum_{k, h=1}^{6}\left\{\frac{1}{36}\left(\Gamma_{D L}^{k b} \Gamma_{D L}^{* k s}\right)\left(\Gamma_{Q L}^{* h q} \Gamma_{Q L}^{h q}\right)\right. \\
& \times\left[G\left(x_{d_{k} g}, x_{q_{h} g}\right)-20 F\left(x_{d_{k} g}, x_{q_{h} g}\right)\right] \\
& +\delta_{q d} \frac{1}{12}\left(\Gamma_{D L}^{k b} \Gamma_{D L}^{* h s}\right)\left(\Gamma_{Q L}^{* k q} \Gamma_{Q L}^{h q}\right) \\
& \left.\times\left[7 G\left(x_{d_{k} g}, x_{q_{h} g}\right)+4 F\left(x_{d_{k} g}, x_{q_{h} g}\right)\right]\right\}
\end{aligned}
$$

\footnotetext{
${ }^{3}$ Note that these diagrams are finite and all the manipulations needed to eliminate the charge conjugation matrices in the crossed topologies shown in Figs. 5 are well defined.
} 


$$
\begin{aligned}
C_{12, \tilde{g}}^{q}\left(\mu_{W}\right)= & \frac{1}{16 \pi^{2}} \frac{1}{m_{\tilde{g}}^{2}} \sum_{k, h=1}^{6}\left\{\frac{1}{12}\left(\Gamma_{D L}^{k b} \Gamma_{D L}^{* k s}\right)\left(\Gamma_{Q L}^{* h q} \Gamma_{Q L}^{h q}\right)\right. \\
& \times\left[7 G\left(x_{d_{k} g}, x_{q_{h} g}\right)+4 F\left(x_{d_{k} g}, x_{q_{h} g} g\right]\right. \\
& +\delta_{q d} \frac{1}{36}\left(\Gamma_{D L}^{k b} \Gamma_{D L}^{* h s}\right)\left(\Gamma_{Q L}^{* k q} \Gamma_{Q L}^{h q}\right) \\
& \left.\times\left[G\left(x_{d_{k} g}, x_{q_{h} g}\right)-20 F\left(x_{d_{k} g}, x_{q_{h} g}\right)\right]\right\} \\
C_{13, \tilde{g}}^{q}\left(\mu_{W}\right)= & \frac{1}{16 \pi^{2}} \frac{1}{m_{\tilde{g}}^{2}} \sum_{k, h=1}^{6}\left\{-\frac{1}{18}\left(\Gamma_{D L}^{k b} \Gamma_{D L}^{* k s}\right)\left(\Gamma_{Q R}^{* h q} \Gamma_{Q R}^{h q}\right)\right. \\
& \times\left[5 G\left(x_{d_{k} g}, x_{q_{h} g}\right)-F\left(x_{d_{k} g}, x_{q_{h} g}\right)\right] \\
& +\delta_{q d} \frac{5}{12}\left(\Gamma_{D L}^{k b} \Gamma_{D L}^{* h s}\right) \\
& \left.\times\left(\Gamma_{Q R}^{* k q} \Gamma_{Q R}^{h q}\right) G\left(x_{d_{k} g}, x_{q_{h} g}\right)\right\} \\
& \left.\times\left(\Gamma_{Q R}^{* k q} \Gamma_{Q R}^{h q}\right) G\left(x_{d_{k} g}, x_{q_{h} g}\right)\right\} \\
C_{14, \tilde{g}}^{q}\left(\mu_{W}\right)= & \frac{1}{16 \pi^{2}} \frac{1}{m_{\tilde{g}}^{2}} \sum_{k, h=1}^{6}\left\{\frac{1}{6}\left(\Gamma_{D L}^{k b} \Gamma_{D L}^{* k s}\right)\left(\Gamma_{Q R}^{* h q} \Gamma_{Q R}^{h q}\right)\right. \\
& \times\left[G\left(x_{d_{k} g}, x_{q_{h} g}\right)+7 F\left(x_{d_{k} g}, x_{q_{h} g}\right)\right] \\
& \delta_{q d} \frac{11}{36}\left(\Gamma_{D L}^{k b} \Gamma_{D L}^{* h s}\right) \\
&
\end{aligned}
$$

with the corresponding primed coefficients obtained through the interchange $\Gamma_{D L}^{i j} \leftrightarrow \Gamma_{D R}^{i j}$ and $\Gamma_{Q L}^{i j} \leftrightarrow \Gamma_{Q R}^{i j}$. Notice that the symbol $\delta_{q d}$ is the Kronecker delta, equal to one when $q$ is the down-quark and zero when $q$ is a different quark. For $q=d$, also, the subscript $Q$ in the two combinations $\left(\Gamma_{Q L}^{* h q} \Gamma_{Q L}^{h q}\right)$ and $\left(\Gamma_{Q R}^{* h q} \Gamma_{Q R}^{h q}\right)$ has to be identified with $D$, typical of a down-type squark exchanged in the box diagram. The boxdiagram functions $G(x, y)$ and $F(x, y)$ are explicitly listed in Appendix A.

The remaining coefficients $C_{15, \tilde{g}}^{q}-C_{20, \tilde{g}}^{q}$, in mass insertion language, are characterized by an odd number of $L-R$ insertions in each squark line. In the mass-eigenstate basis used for squarks in this analysis, they are

$$
\begin{aligned}
C_{15, \tilde{g}}^{q}\left(\mu_{W}\right)= & \frac{1}{16 \pi^{2}} \frac{1}{m_{\tilde{g}}^{2}} \sum_{k, h=1}^{6}\left\{\frac{11}{18}\left(\Gamma_{D R}^{k b} \Gamma_{D L}^{* k s}\right)\right. \\
& \times\left(\Gamma_{Q L}^{* h q} \Gamma_{Q R}^{h q}\right) F\left(x_{d_{k} g}, x_{q_{h} g}\right) \\
& -\delta_{q d} \frac{8}{3}\left(\Gamma_{D R}^{k b} \Gamma_{D L}^{* h s}\right) \\
& \left.\times\left(\Gamma_{Q L}^{* k q} \Gamma_{Q R}^{h q}\right) F\left(x_{d_{k} g}, x_{q_{h} g}\right)\right\}
\end{aligned}
$$

$$
\begin{aligned}
& C_{16, \tilde{g}}^{q}\left(\mu_{W}\right)=\frac{1}{16 \pi^{2}} \frac{1}{m_{\tilde{g}}^{2}} \sum_{k, h=1}^{6}\left\{\frac{5}{6}\left(\Gamma_{D R}^{k b} \Gamma_{D L}^{* k s}\right)\right. \\
& \times\left(\Gamma_{Q L}^{* h q} \Gamma_{Q R}^{h q}\right) F\left(x_{d_{k} g}, x_{q_{h} g}\right) \\
& +\delta_{q d} \frac{4}{9}\left(\Gamma_{D R}^{k b} \Gamma_{D L}^{* h s}\right) \\
& \left.\times\left(\Gamma_{Q L}^{* k q} \Gamma_{Q R}^{h q}\right) F\left(x_{d_{k} g}, x_{q_{h} g}\right)\right\}, \\
& C_{17, \tilde{g}}^{q}\left(\mu_{W}\right)=\frac{1}{16 \pi^{2}} \frac{1}{m_{\tilde{g}}^{2}} \sum_{k, h=1}^{6}\left\{-\frac{11}{18}\left(\Gamma_{D R}^{k b} \Gamma_{D L}^{* k s}\right)\right. \\
& \times\left(\Gamma_{Q R}^{* h q} \Gamma_{Q L}^{h q}\right) G\left(x_{d_{k} g}, x_{q_{h} g}\right) \\
& -\delta_{q d} \frac{1}{3}\left(\Gamma_{D R}^{k b} \Gamma_{D L}^{* h s}\right)\left(\Gamma_{Q R}^{* k q} \Gamma_{Q L}^{h q}\right) \\
& \left.\times\left[G\left(x_{d_{k} g}, x_{q_{h} g}\right)+7 F\left(x_{d_{k} g}, x_{q_{h} g}\right)\right]\right\}, \\
& C_{18, \tilde{g}}^{q}\left(\mu_{W}\right)=\frac{1}{16 \pi^{2}} \frac{1}{m_{\tilde{g}}^{2}} \sum_{k, h=1}^{6}\left\{-\frac{5}{6}\left(\Gamma_{D R}^{k b} \Gamma_{D L}^{* k s}\right)\right. \\
& \times\left(\Gamma_{Q R}^{* h q} \Gamma_{Q L}^{h q}\right) G\left(x_{d_{k} g}, x_{q_{h} g}\right) \\
& +\delta_{q d} \frac{1}{9}\left(\Gamma_{D R}^{k b} \Gamma_{D L}^{* h s}\right)\left(\Gamma_{Q R}^{* k q} \Gamma_{Q L}^{h q}\right) \\
& \left.\times\left[5 G\left(x_{d_{k} g}, x_{q_{h} g}\right)-F\left(x_{d_{k} g}, x_{q_{h} g}\right)\right]\right\}, \\
& C_{19, \tilde{g}}^{q}\left(\mu_{W}\right)=\frac{1}{16 \pi^{2}} \frac{1}{m_{\tilde{g}}^{2}} \sum_{k, h=1}^{6}\left\{-\frac{1}{8}\left(\Gamma_{D R}^{k b} \Gamma_{D L}^{* k s}\right)\right. \\
& \times\left(\Gamma_{Q L}^{* h q} \Gamma_{Q R}^{h q}\right) F\left(x_{d_{k} g}, x_{q_{h} g}\right) \\
& +\delta_{q d} \frac{1}{12}\left(\Gamma_{D R}^{k b} \Gamma_{D L}^{* h s}\right) \\
& \left.\times\left(\Gamma_{Q L}^{* k q} \Gamma_{Q R}^{h q}\right) F\left(x_{d_{k} g}, x_{q_{h} g}\right)\right\}, \\
& C_{20, \tilde{g}}^{q}\left(\mu_{W}\right)=\frac{1}{16 \pi^{2}} \frac{1}{m_{\tilde{g}}^{2}} \sum_{k, h=1}^{6}\left\{\frac{3}{8}\left(\Gamma_{D R}^{k b} \Gamma_{D L}^{* k s}\right)\right. \\
& \times\left(\Gamma_{Q L}^{* h q} \Gamma_{Q R}^{h q}\right) F\left(x_{d_{k} g}, x_{q_{h} g}\right) \\
& -\delta_{q d} \frac{5}{36}\left(\Gamma_{D R}^{k b} \Gamma_{D L}^{* h s}\right) \\
& \left.\times\left(\Gamma_{Q L}^{* k q} \Gamma_{Q R}^{h q}\right) F\left(x_{d_{k} g}, x_{q_{h} g}\right)\right\} .
\end{aligned}
$$

The considerations made for the coefficients (32) hold also here: the corresponding primed coefficients are obtained 
through the interchanges $\Gamma_{D L}^{i j} \leftrightarrow \Gamma_{D R}^{i j}$ and $\Gamma_{Q L}^{i j} \leftrightarrow \Gamma_{Q R}^{i j}$, and $\delta_{q d}$ always vanishes, except for $q=d$. Under renormalization, the operators corresponding to the coefficients (33) mix with the magnetic and chromomagnetic operators in Eqs. (11) and (13) by undergoing a chirality flip proportional to $m_{q}$. Therefore, only $q=b$ and $q=c$ can contribute to the decay $b \rightarrow s \gamma$ in the approximation of massless light quarks made here.

\section{WILSON COEFFICIENTS AT THE DECAY SCALE}

As already mentioned in Sec. II, the two terms $\mathcal{H}_{\text {eff }}^{W}$ and $\mathcal{H}_{\text {eff }}^{\tilde{g}}$ in the effective Hamiltonian (9) undergo separate renormalization. The anomalous-dimension matrix of the SM operators $\mathcal{O}_{1}-\mathcal{O}_{8}$ and the evolution of the corresponding Wilson coefficients to the decay scale $\mu_{b}$ are very well known and can be found in [23].

The evolution of the gluino-induced Wilson coefficients $C_{i, \tilde{g}}$ from the matching scale $\mu_{W}$ down to the low-energy scale $\mu_{b}$ is described by the renormalization group equation:

$$
\mu \frac{d}{d \mu} C_{i, \tilde{g}}=C_{j, \tilde{g}}(\mu) \gamma_{j i, \tilde{g}}(\mu)
$$

The usual perturbative expansion for the initial conditions of the Wilson coefficients,

$$
C_{i, \tilde{g}}\left(\mu_{W}\right)=C_{i, \tilde{g}}^{0}\left(\mu_{W}\right)+\frac{\alpha_{s}\left(\mu_{W}\right)}{4 \pi} C_{i, \tilde{g}}^{1}\left(\mu_{W}\right)+\cdots
$$

as well as for the elements of $\gamma_{j i} \tilde{g}(\mu)$,

$$
\gamma_{j i, \tilde{g}}(\mu)=\frac{\alpha_{s}(\mu)}{4 \pi} \gamma_{j i, \tilde{g}}^{0}+\frac{\alpha_{s}^{2}(\mu)}{(4 \pi)^{2}} \gamma_{j i, \tilde{g}}^{1}+\cdots,
$$

is possible thanks to the choice of including appropriate powers of $g_{s}(\mu)$ into the definition of the operators $\mathcal{O}_{i, \tilde{g}}$, as discussed in Sec. II. Since no NLO results are presented in this paper, the symbol $\gamma_{j i, \tilde{g}}(\mu)$ will be used in the following to indicate the LO quantity $\gamma_{j i, \tilde{g}}^{0}(\mu)$. Similarly the Wilson coefficients $C_{i, \tilde{g}}$ will be indicating $C_{i, \tilde{g}}^{0}$, as already understood in the previous sections. The indices $i, j$ in Eqs. (35) and (36) run over all gluino-induced operators: 12 magnetic and chromomagnetic operators and 5 times (one for each flavor q) 20 four-quark operators. The anomalous-dimension matrix $\gamma_{j i, \tilde{g}}$ is then a $112 \times 112$ matrix. It turns out, however, that primed and non-primed operators do not mix. This reduces the problem to the evaluation of two identical 56 $\times 56$ matrices.

Moreover, given their lower dimensionality, the dimension-five operators $\mathcal{O}_{7 \tilde{g}, \tilde{g}}, \mathcal{O}_{8, \tilde{g}, \tilde{g}}$, and $\mathcal{O}_{7 \tilde{g}, \tilde{g}}^{\prime}, \mathcal{O}_{8 \tilde{g}, \tilde{g}}^{\prime}$, do not mix with dimension-six magnetic operators. The 4 $\times 4$ submatrix for these operators is a block-diagonal matrix with $2 \times 2$ blocks. The block corresponding to $\mathcal{O}_{7 \tilde{g}, \tilde{g}}, \mathcal{O}_{8} \tilde{g}, \tilde{g}$ is

$$
\gamma_{j i, \tilde{g}}=\left[\begin{array}{cc}
18 & 0 \\
-\frac{32}{9} & \frac{50}{3}
\end{array}\right](i, j=7 \tilde{g}, 8 \tilde{g}) \text {, }
$$

and differs from the known mixing matrix of the SM operators $\mathcal{O}_{7}$ and $\mathcal{O}_{8}$ just by anomalous dimensions of the explicit mass $\bar{m}_{b}$ and of the coupling $g_{s}^{2}$ in the definition of the operators.

In general, the structure of the remaining $54 \times 54$ matrix, corresponding to the four-quark operators $\mathcal{O}_{i, \tilde{g}}^{q} \quad(i$ $=11, \ldots, 20 ; q=u, d, c, s, b)$, magnetic operators $\mathcal{O}_{7 b, \tilde{g}}$, $\mathcal{O}_{7 c, \tilde{g}}$, and the chromomagnetic operators $\mathcal{O}_{8 b, \tilde{g}}, \mathcal{O}_{8 c, \tilde{g}}$, is rather complicated. The fact that in a LO calculation only the coefficients $C_{7 b, \tilde{g}}$ and $C_{7 c, \tilde{g}}$ (and corresponding primed coefficients) are needed at the low scale $\mu_{b}$, however, simplifies the analysis considerably. Among the four-quark operators, only those with scalar-tensor Lorentz structure, i.e. $\mathcal{O}_{i, \tilde{g}}^{q}$ $(i=15, \ldots, 20)$, mix into the magnetic and chromomagnetic operators at order $\alpha_{s}$. The vector operators $\left[\mathcal{O}_{i, \tilde{g}}^{q}(i\right.$ $=11, \ldots, 14)]$ on the other hand mix neither into the magnetic and chromomagnetic operators nor into the scalartensor four-quark operators. (The scalar-tensor operators, however, mix into the vector four-quark operators.) This implies that the presence of the four-quark operators with vector structure is completely irrelevant for the evolution of the coefficients of the magnetic operators. The observation that the scalar-tensor operators with the label $q$ mix into $\mathcal{O}_{7 q, \tilde{g}}$ and $\mathcal{O}_{8 q, \tilde{g}}$, with the same $q$, together with the fact that scalar-tensor operators mix among themselves in a flavordiagonal way, further simplifies the situation. It is indeed possible to restrict the problem at the LO level to the calculation of two $8 \times 8$ matrices, i.e. the two matrices corresponding to the operators $\mathcal{O}_{15, \tilde{g}}^{q}, \mathcal{O}_{16, \tilde{g}}^{q}, \quad \mathcal{O}_{17, \tilde{g}}^{q}, \mathcal{O}_{18, \tilde{g}}^{q}$, $\mathcal{O}_{19, \tilde{g}}^{q}, \mathcal{O}_{20, \tilde{g}}^{q}, \mathcal{O}_{7 q, \tilde{g}}, \mathcal{O}_{8 q, \tilde{g}}$, for $q=b$ and $q=c$.

For the case $q=b$, the result of such a calculation, in which the anomalous dimensions due to the explicit powers of the coupling $\alpha_{s}$ are again included, is 


$$
\left\{\gamma_{j i, \tilde{g}}\right\}=\left[\begin{array}{rrrrrrrr}
\frac{44}{3} & 0 & 0 & 0 & \frac{1}{3} & -1 & -\frac{1}{3} & 1 \\
-6 & \frac{98}{3} & 0 & 0 & -\frac{1}{2} & -\frac{7}{6} & -1 & 0 \\
0 & 0 & \frac{44}{3} & 0 & 0 & 0 & 0 & 0 \\
0 & 0 & -6 & \frac{98}{3} & 0 & 0 & 0 & 0 \\
16 & -48 & 0 & 0 & 36 & 0 & \frac{28}{3} & -4 \\
-24 & -56 & 0 & 0 & 6 & 18 & \frac{20}{3} & -8 \\
0 & 0 & 0 & 0 & 0 & 0 & 26 & 0 \\
0 & 0 & 0 & 0 & 0 & 0 & -\frac{32}{9} & \frac{74}{3}
\end{array}\right] .
$$

The anomalous-dimension matrix corresponding to the case $q=c$ differs from the previous one in the submatrix responsible for mixing of the four-quark operators into the magnetic and chromomagnetic operators:

$$
\left\{\gamma_{j i, \tilde{g}}\right\}=\left[\begin{array}{rrrrrrrr}
\frac{44}{3} & 0 & 0 & 0 & \frac{1}{3} & -1 & 0 & 0 \\
-6 & \frac{98}{3} & 0 & 0 & -\frac{1}{2} & -\frac{7}{6} & 0 & 0 \\
0 & 0 & \frac{44}{3} & 0 & 0 & 0 & 0 & 0 \\
0 & 0 & -6 & \frac{98}{3} & 0 & 0 & 0 & 0 \\
16 & -48 & 0 & 0 & 36 & 0 & -16 & 0 \\
-24 & -56 & 0 & 0 & 6 & 18 & -\frac{16}{3} & -8 \\
0 & 0 & 0 & 0 & 0 & 0 & 26 & 0 \\
0 & 0 & 0 & 0 & 0 & 0 & -\frac{32}{9} & \frac{74}{3}
\end{array}\right]
$$

Using the anomalous dimensions matrices (37), (38) and (39), the renormalization group equation (34) can be solved by the standard procedure, described, for example, in Ref. [40], using the Wilson coefficients $C_{i, \tilde{g}}\left(\mu_{W}\right)$ given in Sec. IV as initial conditions. The integration of Eq. (34) for $C_{7} \tilde{g}, \tilde{g}$ and $C_{8 \tilde{g}, \tilde{g}}$ yields the following expressions for these Wilson coefficients at the low scale $\mu_{b}$ :

$$
\begin{aligned}
C_{7 \tilde{g}, \tilde{g}}\left(\mu_{b}\right)= & \eta^{27 / 23} C_{7 \tilde{g}, \tilde{g}}\left(\mu_{W}\right) \\
& +\frac{8}{3}\left(\eta^{25 / 23}-\eta^{27 / 23}\right) C_{8 \tilde{g}, \tilde{g}}\left(\mu_{W}\right),
\end{aligned}
$$

$$
C_{8 \tilde{g}, \tilde{g}}\left(\mu_{b}\right)=\eta^{25 / 23} C_{8 \tilde{g}, \tilde{g}}\left(\mu_{W}\right)
$$

Here and in the following, $\eta$ denotes the ratio $\alpha_{s}\left(\mu_{W}\right) / \alpha_{s}\left(\mu_{b}\right)$. The low-scale Wilson coefficients for the corresponding primed operators are obtained by replacing in Eq. (40) all the unprimed coefficients with primed ones. The same holds for the following coefficients.

The Wilson coefficients of the dimension-six operators $C_{7 b, \tilde{g}}$ and $C_{8 b, \tilde{g}}$ are at low scale: 


$$
\begin{aligned}
C_{7 b, \tilde{g}}\left(\mu_{b}\right)= & \eta^{39 / 23} C_{7 b, \tilde{g}}\left(\mu_{W}\right) \\
& +\frac{8}{3}\left(\eta^{37 / 23}-\eta^{39 / 23}\right) C_{8 b, \tilde{g}}\left(\mu_{W}\right) \\
& +R_{7 b, \tilde{g}}\left(\mu_{b}\right), \\
C_{8 b, \tilde{g}}\left(\mu_{b}\right)= & \eta^{\frac{37}{23}} C_{8 b, \tilde{g}}\left(\mu_{W}\right)+R_{8 b, \tilde{g}}\left(\mu_{b}\right) .
\end{aligned}
$$

The remainder functions $R_{7 b, \tilde{g}}\left(\mu_{b}\right)$ and $R_{8 b, \tilde{g}}\left(\mu_{b}\right)$ are given in Appendix B. They turn out to be numerically very small with respect to the other terms on the right-hand sides of Eq. (41). Notice that, in the approximation $R_{7 b, \tilde{g}}\left(\mu_{b}\right)$ $=R_{8 b, \tilde{g}}\left(\mu_{b}\right)=0$, the low-scale coefficients $C_{7 b, \tilde{g}}\left(\mu_{b}\right)$ and $C_{8 b, \tilde{g}}\left(\mu_{b}\right)$ are simply obtained through the integration of Eq. (34) with the anomalous dimension matrix $\gamma_{j i, \tilde{g}}$ reduced to the $2 \times 2$ block of Eq. (38) corresponding to the operators $\mathcal{O}_{7 b, \tilde{g}}$ and $\mathcal{O}_{8 b, \tilde{g}}$.

Finally, the coefficients $C_{7 c, \tilde{g}}\left(\mu_{b}\right)$ and $C_{8 c, \tilde{g}}\left(\mu_{b}\right)$ formally have the same expression as $C_{7 b, \tilde{g}}\left(\mu_{b}\right)$ and $C_{8 b, \tilde{g}}\left(\mu_{b}\right)$, when the indices $7 b$ and $8 b$ are replaced by $7 c$ and $8 c$. Also in this case, the functions $R_{7 c, \tilde{g}}\left(\mu_{b}\right)$ and $R_{8 c, \tilde{g}}\left(\mu_{b}\right)$, listed in Appendix B, are numerically small. In the approximation $R_{7 c, \tilde{g}}\left(\mu_{b}\right)=R_{8 c, \tilde{g}}\left(\mu_{b}\right)=0$, the coefficients $C_{7 c, \tilde{g}}\left(\mu_{b}\right)$ and $C_{8 c, \tilde{g}}\left(\mu_{b}\right)$ vanish identically, since the corresponding Wilson coefficients at the matching scale are vanishing.

\section{BRANCHING RATIO}

The branching ratio $\mathrm{BR}\left(\bar{B} \rightarrow X_{s} \gamma\right)$ can be expressed as

$$
\operatorname{BR}\left(\bar{B} \rightarrow X_{s} \gamma\right)=\frac{\Gamma(b \rightarrow s \gamma)}{\Gamma_{S L}} \mathrm{BR}_{S L}
$$

where $\mathrm{BR}_{S L}=(10.49 \pm 0.46) \%$ is the measured semileptonic branching ratio. To the relevant order in $\alpha_{s}$, the semileptonic decay width is given by

$$
\Gamma_{S L}=\frac{m_{b}^{5} G_{F}^{2}\left|V_{c b}\right|^{2}}{192 \pi^{3}} g\left(\frac{m_{c}^{2}}{m_{b}^{2}}\right)
$$

where the phase-space function $g(z)$ is $g(z)=1-8 z+8 z^{3}$ $-z^{4}-12 z^{2} \log z$. The decay width for $b \rightarrow s \gamma$ reads

$$
\Gamma(b \rightarrow s \gamma)=\frac{m_{b}^{5} G_{F}^{2}\left|V_{t b} V_{t s}^{*}\right|^{2} \alpha}{32 \pi^{4}}\left(\left|\hat{C}_{7}\right|^{2}+\left|\hat{C}_{7}^{\prime}\right|^{2}\right)
$$

where $\hat{C}_{7}$ and $\hat{C}_{7}^{\prime}$ can be expressed in terms of the SM and gluino-induced Wilson coefficients evolved down to the decay scale $\mu_{b}$ as

$$
\begin{aligned}
\hat{C}_{7}= & -\frac{16 \sqrt{2} \pi^{3} \alpha_{s}\left(\mu_{b}\right)}{G_{F} V_{t b} V_{t s}^{*}}\left[C_{7 b, \tilde{g}}\left(\mu_{b}\right)+\frac{1}{m_{b}} C_{7 \tilde{g}, \tilde{g}}\left(\mu_{b}\right)\right. \\
& \left.+\frac{m_{c}}{m_{b}} C_{7 c, \tilde{g}}\left(\mu_{b}\right)\right]+C_{7}\left(\mu_{b}\right),
\end{aligned}
$$

$$
\begin{aligned}
\hat{C}_{7}^{\prime}= & -\frac{16 \sqrt{2} \pi^{3} \alpha_{s}\left(\mu_{b}\right)}{G_{F} V_{t b} V_{t s}^{*}}\left[C_{7 b, \tilde{g}}^{\prime}\left(\mu_{b}\right)+\frac{1}{m_{b}} C_{7, \tilde{g}, \tilde{g}}^{\prime}\left(\mu_{b}\right)\right. \\
& \left.+\frac{m_{c}}{m_{b}} C_{7 c, \tilde{g}}^{\prime}\left(\mu_{b}\right)\right] .
\end{aligned}
$$

Notice that, at the leading logarithmic level, it is not possible to distinguish between the pole masses $m_{b}$ and $m_{c}$ from the corresponding running quantities at the scale $m_{b}$ or $m_{c}$. In the following, these mass parameters are always treated as pole masses.

\section{NUMERICAL RESULTS}

Numerical predictions for the QCD-corrected branching ratio $\operatorname{BR}\left(\bar{B} \rightarrow X_{s} \gamma\right)$ induced by gluino-squark exchange can be obtained from Eqs. (42)-(45). To show these results, it is convenient to select one possible source of flavor violation in the squark sector at a time and assume that all the remaining ones are vanishing.

Following Ref. [28], all diagonal entries in $m_{d, L L}^{2}$, $m_{d, R R}^{2}$, and $m_{u, R R}^{2}$ are set to be equal and their common value is denoted by $m_{\tilde{q}}^{2}$. The branching ratio can then be studied as a function of only one off-diagonal element in $m_{d, L L}^{2}$ and $m_{d, R R}^{2}$, normalized to $m_{\tilde{q}}^{2}$, i.e. as a function of one of the elements

$$
\delta_{L L, i j}=\frac{\left(m_{d, L L}^{2}\right)_{i j}}{m_{\tilde{q}}^{2}}, \quad \delta_{R R, i j}=\frac{\left(m_{d, R R}^{2}\right)_{i j}}{m_{\tilde{q}}^{2}}, \quad(i \neq j)
$$

and/or of one diagonal or off-diagonal element of the $3 \times 3$ matrices $m_{d, L R}^{2}, m_{d, R L}^{2}$ again normalized to $m_{\tilde{q}}^{2}$ :

$$
\delta_{L R, i j}=\frac{\left(m_{d, L R}^{2}\right)_{i j}}{m_{\tilde{q}}^{2}}, \quad \delta_{R L, i j}=\frac{\left(m_{d, R L}^{2}\right)_{i j}^{\dagger}}{m_{\tilde{q}}^{2}} .
$$

The corresponding off-diagonal entries in the up-squark mass matrix squared, relevant for the contributions coming from the gluino-induced four-quark operators (14) and (15) are set to be equal to those in the down-squark mass matrix squared. Among the four-quark operators, only the scalartensor operators (15) contribute to $\mathrm{BR}\left(\bar{B} \rightarrow X_{s} \gamma\right)$, at LO in QCD. Their effect is negligible and the above restriction is not likely to produce an unnatural reduction of their contribution. Indeed, due to their proportionality to $\Gamma_{D R}^{k b} \Gamma_{D L}^{* k s}$, the operators $\mathcal{O}_{i, \tilde{g}}^{q}(i=15, \ldots, 20)$ are generated always together with $\mathcal{O}_{7 \tilde{g}, \tilde{g}}$ and $\mathcal{O}_{8, \tilde{g}, \tilde{g}}$. As will be discussed later, the latter are the numerically important operators and the corrections induced, e.g., by $\mathcal{O}_{8} \tilde{g}, \tilde{g}$ on the Wilson coefficent $C_{7 \tilde{g}, \tilde{g}}\left(\mu_{b}\right)$ completely overshadow the effect of the four-quark operators $\mathcal{O}_{i, \tilde{g}}^{q}$. These induce corrections of the Wilson coefficient $C_{7 \tilde{b}, \tilde{g}}\left(\mu_{b}\right)$ of the numerically less relevant operator $\mathcal{O}_{7 \tilde{b}, \tilde{g}}$ that are generically suppressed by a factor $\left(m_{b} / m_{g}\right)$ at the amplitude level. Analogously, the primed scalar-tensor operators $\mathcal{O}_{i, g}^{q^{\prime}}(i=15, \ldots, 20)$ are also expected to have a very small impact on the decay amplitude. The vector four-quark 
operators, on the other hand, can be generated without the simultaneous generation of $\mathcal{O}_{7 \tilde{g}, \tilde{g}}$ and $\mathcal{O}_{8 \tilde{g}, \tilde{g}}$ and no suppression factor $\left(m_{b} / m_{\tilde{g}}\right)$ is present in this case. Therefore, the vector four-quark operators, although entering at NLO only, are in general expected to have a larger impact on the decay amplitude than the scalar-tensor four-quark operators. In the context of a NLO analysis, one should actually check if the assumption of equal off-diagonal entries in the up- and down-squark mass matrices squared is not an oversimplification, affecting the generality of the numerical results.

As for the remaining entries in the squark mass matrices squared, the $D$ terms are calculated using $M_{Z}=91.18 \mathrm{GeV}$, $\sin ^{2} \theta_{W}=0.2316$, and $\tan \beta=2$; the $F$ terms $F_{f L L}$ and $F_{f R R}$, using $m_{b}=3 \mathrm{GeV}$ and $m_{t}=175 \mathrm{GeV}$, in the approximation of vanishing lighter quark masses, whereas $F_{f L R}=F_{f R L}=0$ is assumed. It is obvious that all the information gained through the numerical evaluation of $\operatorname{BR}\left(\bar{B} \rightarrow X_{s} \gamma\right)$ on the size of $\left(m_{d, L R}^{2}\right)_{33}$ can be extended to the combination $\left(m_{d, L R}^{2}+F_{f L R}\right)_{33}$ and $\left[\left(m_{d, L R}^{2}\right)^{\dagger}+F_{f R L}\right]_{33}$ in realistic cases, in which $\mu \neq 0$. For the diagonal entry $m_{\tilde{q}}^{2}$, the value $m_{q}=500 \mathrm{GeV}$ is in general used. Moreover, it is imposed that the eigenvalues of the two $6 \times 6$ up- and down-squark mass matrices are larger than $150 \mathrm{GeV}$ for all values of the $\delta$ ratios scanned. The value of $150 \mathrm{GeV}$ is here taken as an average model-independent lower limit on squark masses, which can be inferred from direct searches of squarks at hadron colliders.

Finally, the remaining parameter needed to determine the branching ratio is

$$
x=\frac{m_{\tilde{g}}^{2}}{m_{\tilde{q}}^{2}},
$$

where $m_{g}$ is the gluino mass.

In the following, the $\mathrm{SM}$ contribution to $\operatorname{BR}\left(\bar{B} \rightarrow X_{s} \gamma\right)$ is, in general, added to the gluino contribution: possible constraints on the flavor-violating sources in the squark sector should be extracted, keeping into account that the SM contribution already successfully saturates the experimental result for this branching ratio $[12,13]$. As already stressed in Sec. I, this analysis applies to particular directions of the supersymmetric parameter space, in which charged Higgs, chargino, and neutralino contributions can be safely neglected with respect to the gluino and SM contributions. Moreover, it should also be mentioned that the bounds discussed in this section on $\delta_{L L, 23}, \delta_{R R, 23}, \delta_{L R, 23}$, and $\delta_{R L, 23}$, obtained in these particular directions of parameter space, have to be understood in an indicative sense, since they are extracted ignoring the error of the theoretical calculation.

It is useful to isolate the gluino contribution when illustrating the impact of the LO QCD corrections on the gluinoinduced Hamiltonian. In Figs. 6 and 7, indicated by solid lines, are shown the values of the QCD-corrected branching ratio obtained, respectively, when only $\delta_{L R, 23}$ and $\delta_{L L, 23}$ are non-vanishing. Their values are fixed in the two figures as follows: $\delta_{L R, 23}=0.01$ and $\delta_{L L, 23}=0.5$. The branching ratio is plotted as a function of $x$, i.e. as a function of the gluino

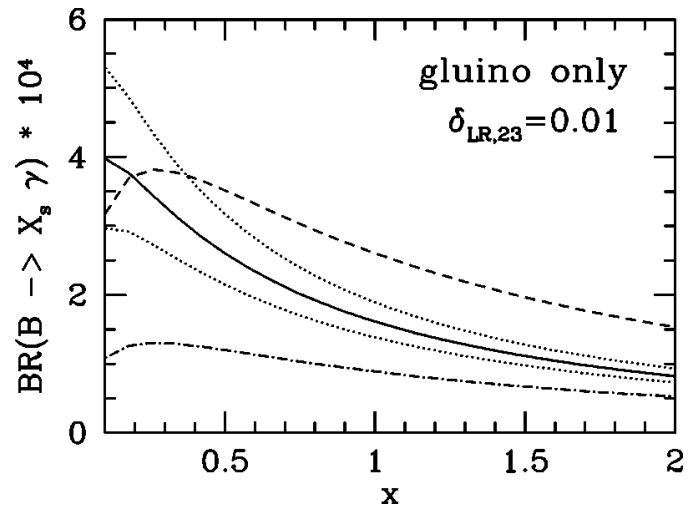

FIG. 6. Gluino-induced branching ratio $\mathrm{BR}\left(\bar{B} \rightarrow X_{s} \gamma\right)$ as a function of $x=m_{\tilde{g}}^{2} / m_{\tilde{q}}^{2}$, obtained when the only source of flavor violation is $\delta_{L R, 23}$ (see text), fixed to the value 0.01 , for $m_{q}^{\sim}=500 \mathrm{GeV}$. The solid line shows the branching ratio at the LO in QCD, for $\mu_{b}=4.8 \mathrm{GeV}$ and $\mu_{W}=M_{W}$; the two dotted lines indicate the range of variation of the branching ratio when $\mu_{b}$ spans the interval 2.4$9.6 \mathrm{GeV}$. Also shown are the values of $\mathrm{BR}\left(\bar{B} \rightarrow X_{s} \gamma\right)$ when no QCD corrections are included and the explicit factor $\alpha_{s}(\mu)$ in the gluinoinduced operators is evaluated at $4.8 \mathrm{GeV}$ (dashed line) or at $M_{W}$ (dot-dashed line).

mass, for a given value of $m_{\tilde{q}}, m_{\tilde{q}}=500 \mathrm{GeV}$. Also shown is the range of variation of the branching ratio, delimited by dotted lines, obtained when the low-energy scale $\mu_{b}$ spans the interval $2.4-9.6 \mathrm{GeV}$. The matching scale $\mu_{W}$ is here fixed to $M_{W}$. As can be seen, the theoretical estimate of $\operatorname{BR}\left(\bar{B} \rightarrow X_{s} \gamma\right)$ is still largely uncertain $(\sim \pm 25 \%)$. An extraction of bounds on $\delta_{L L, 23}$ and $\delta_{L R, 23}$ more precise than just an order of magnitude would require, therefore, the inclusion of NLO QCD corrections. It should be noticed, however, that the inclusion of corrections at the LO has already removed the large ambiguity on the value to be assigned to the factor $\alpha_{s}(\mu)$ in the gluino-induced magnetic operators (11)-(13). Before adding QCD corrections, the scale in this factor can assume all values from $\mu_{b}$ to $\mu_{W}$. The corresponding values for $\operatorname{BR}\left(\bar{B} \rightarrow X_{s} \gamma\right)$ for the two extreme choices of $\mu$ are indicated in Figs. 6 and 7 by the dot-dashed lines $\left(\mu=M_{W}\right)$ and the dashed lines $(\mu=4.8 \mathrm{GeV})$ : the branching ratio is virtually unknown. The choice $\mu=M_{W}$ gives values for the non-

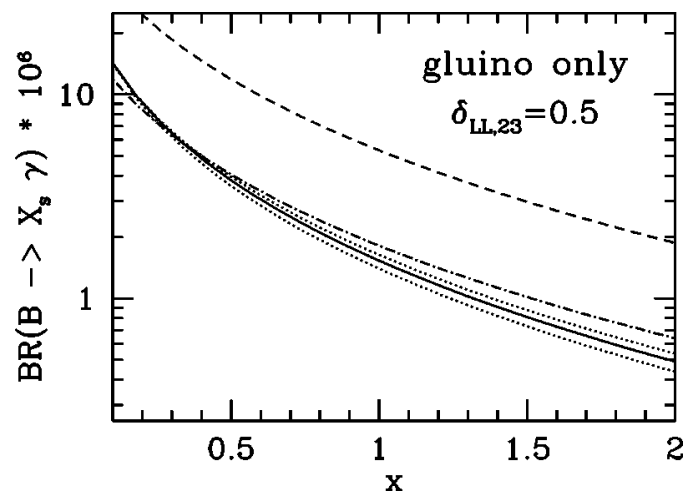

FIG. 7. Same as in Fig. 6 when only $\delta_{L L, 23}$ is non-vanishing and fixed to the value 0.5 . 


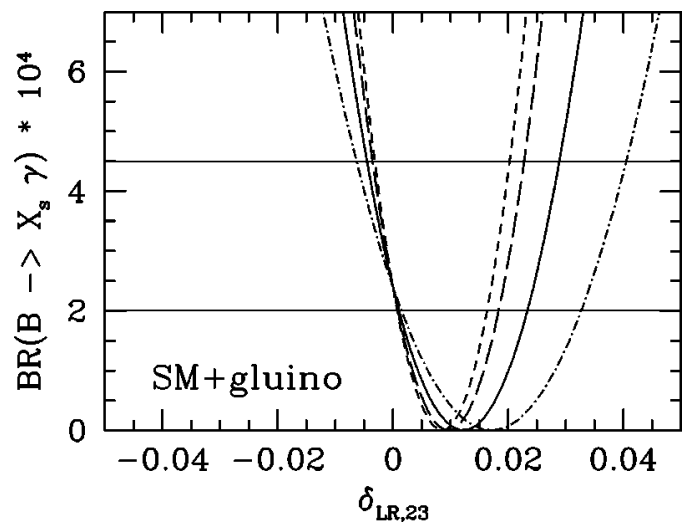

FIG. 8. Dependence of the QCD-corrected branching ratio $\operatorname{BR}\left(\bar{B} \rightarrow X_{s} \gamma\right)$, obtained from the SM and gluino contributions, on the parameter $\delta_{L R, 23}$, when $\left(m_{d, L R}^{2}\right)_{23}$ is the only non-vanishing off-diagonal element in the down-squark mass matrix squared. The branching ratio is shown for different values of $x=m_{\tilde{g}}^{2} / m_{\tilde{q}}^{2}$, with $m_{q}=500 \mathrm{GeV}$ : 0.3 (short-dashed line), 0.5 (long-dashed line), 1 (solid line), and 2 (dot-dashed line). Low and matching scales are $\mu_{b}=4.8 \mathrm{GeV}$ and $\mu_{W}=M_{W}$.

QCD-corrected $\operatorname{BR}\left(\bar{B} \rightarrow X_{s} \gamma\right)$ relatively close to the band obtained for the QCD-corrected result, in the case shown in Fig. 7, when only $\delta_{L L, 23}$ is non-vanishing. Finding a corresponding value of $\mu$ that minimizes the QCD corrections in the case studied in Fig. 6, when only $\delta_{L R, 23}$ is different from zero, depends strongly on the value of $x$.

The results in Figs. 6 and 7 also show that the operator $\mathcal{O}_{7 b, \tilde{g}}$ gives much smaller contributions to $\operatorname{BR}\left(\bar{B} \rightarrow X_{s} \gamma\right)$ than the operator $\mathcal{O}_{7 \tilde{g}, \tilde{g}}$. Indeed, the branching ratio obtained through $\mathcal{O}_{7 b, \tilde{g}}$ only is typically suppressed by a factor $\left(m_{b} / m_{\tilde{g}}\right)^{2}$, with respect to that obtained from $\mathcal{O}_{7 \tilde{g}, \tilde{g}}$, if similar values of $\delta_{L L, 23}$ and $\delta_{L R, 23}$ are chosen. Analogous considerations hold for $\mathcal{O}_{7 b, \tilde{g}}^{\prime}$ and $\mathcal{O}_{7 \tilde{g}, \tilde{g}}^{\prime}$. The elements $\delta_{L R, 23}$ and $\delta_{R L, 23}$ are therefore expected to be the flavor-violating parameters most efficiently constrained by the measurement of $\operatorname{BR}\left(\bar{B} \rightarrow X_{s} \gamma\right)$.

In Fig. 8, the dependence of $\operatorname{BR}\left(\bar{B} \rightarrow X_{s} \gamma\right)$ is shown as a function of $\delta_{L R, 23}$ when this is the only flavor-violating source. The two horizontal lines correspond to the minimum and maximum values, $2 \times 10^{-4}$ and $4.5 \times 10^{-4}$, allowed by the CLEO measurement. The branching ratio is obtained by adding the SM and the gluino contribution calculated for different choices of $x$, and a fixed value of $m_{\tilde{q}}: m_{\tilde{q}}$ $=500 \mathrm{GeV}$. The values of the gluino mass corresponding to the choices $x=0.3,0.5,1,2$ are $m_{g}=274,354,500$, $707 \mathrm{GeV}$. The branching ratio is plotted in this figure for fixed values of the two scales: $\mu_{b}=4.8 \mathrm{GeV}$ and $\mu_{W}$ $=M_{W}$. The gluino contribution interferes constructively with the SM for negative values of $\delta_{L R, 23}$, which are then more sharply constrained than the positive values. Overall, this parameter cannot exceed the per cent level. No interference with the SM is present when $\delta_{R L, 23}$ is the only source of flavor violation, as shown in Fig. 9. The results obtained for $\operatorname{BR}\left(\bar{B} \rightarrow X_{s} \gamma\right)$ are then symmetric around $\delta_{R L, 23}=0$ and the constraints on $\left|\delta_{R L, 23}\right|$ are upper bounds on its absolute

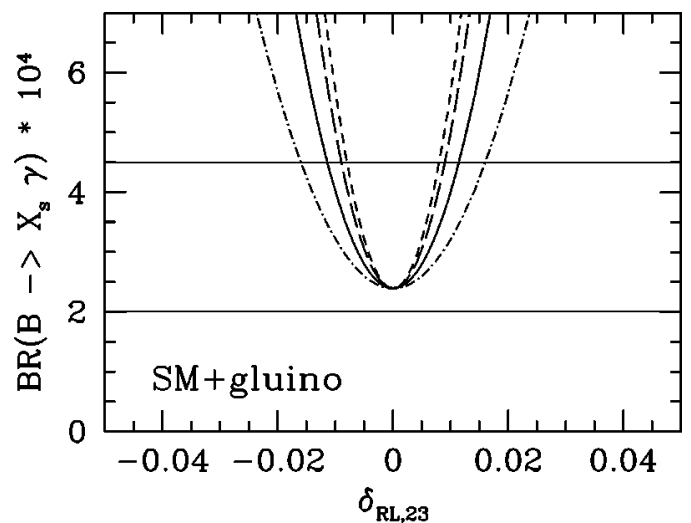

FIG. 9. Same as in Fig. 8, when $\delta_{R L, 23}$ is the only source of flavor violation for the gluino contribution. The parameter $x$ is fixed to 0.3 (short-dashed line), 0.5 (long-dashed line), 1 (solid line), 2 (dot-dashed line).

value: there are no small values of $\delta_{R L, 23}$ for which the total branching ratio falls off the band allowed by the CLEO measurement.

Much weaker is the dependence of $\operatorname{BR}\left(\bar{B} \rightarrow X_{s} \gamma\right)$ on $\delta_{L L, 23}$ if $\left(m_{d, L L}^{2}\right)_{23}$ is the only off-diagonal element in the down squark mass matrix squared. This dependence is illustrated in Fig. 10 for different choices of $x$ and $m_{\tilde{q}}$ $=500 \mathrm{GeV}$. The gluino-squark loop generates in this case only the dimension-six operator $\mathcal{O}_{7 b, \tilde{g}}$ and the gluino contribution interferes constructively with the SM contribution for positive $\delta_{L L, 23}$. Notice that the mass insertion approximation, given the large values of $\delta_{L L, 23}$ allowed by the experimental measurement, cannot be used in this case to obtain a reliable estimate of $\operatorname{BR}\left(\bar{B} \rightarrow X_{s} \gamma\right)$, whereas it is an excellent approximation of the complete calculation in the cases shown in Figs. 8 and 9. For completeness, also the case in which the only off-diagonal element in the down-squark mass matrix squared is in the right-right sector, $\left(m_{d, R R}^{2}\right)_{23}$ $\neq 0$, is shown in Fig. 11. The inclusive branching ratio, plotted versus the relevant parameter $\delta_{R R, 23}$, is now obtained from the incoherent sum of the SM and gluino contributions

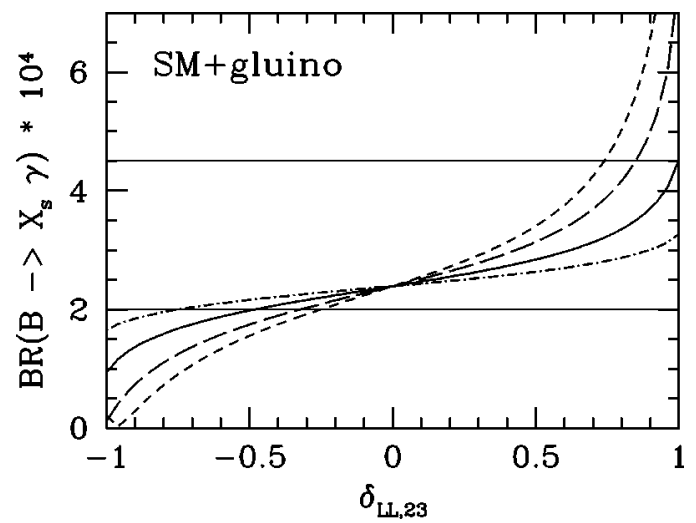

FIG. 10. Same as in Fig. 8, when $\delta_{L L, 23}$ is the only source of flavor violation for the gluino contribution. The different lines correspond to: $x=0.3$ (short-dashed line), 0.5 (long-dashed line), 1 (solid line), 2 (dot-dashed line). 


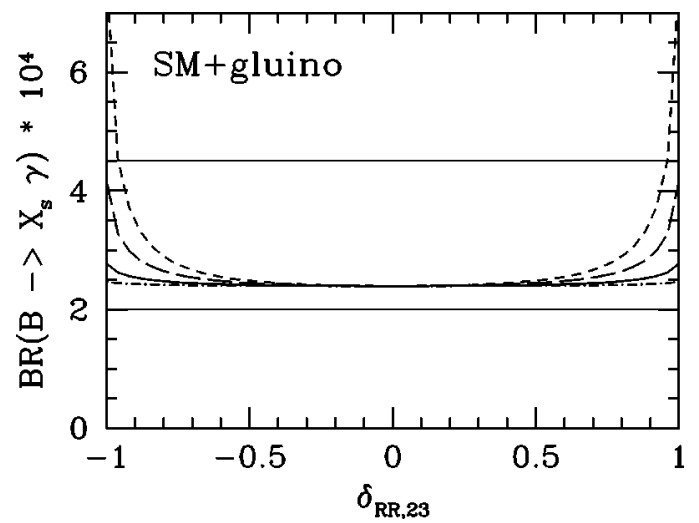

FIG. 11. Same as in Fig. 8, when $\delta_{R R, 23}$ is the only source of flavor violation for the gluino contribution. The values of $x$ corresponding to the different lines are: 0.3 (short-dashed line), 0.5 (long-dashed line), 1 (solid line), 2 (dot-dashed line).

and shows conspicuous deviation from the SM result only for very large values of $\delta_{R R, 23}$.

As already observed, among the operators $\mathcal{O}_{7 b, \tilde{g}}$ and $\mathcal{O}_{7 \tilde{g}, \tilde{g}}$, the second one has the stronger impact on $\operatorname{BR}(\bar{B}$ $\left.\rightarrow X_{s} \gamma\right)$. It is then legitimate to question whether $\mathcal{O}_{7 \tilde{g}, \tilde{g}}$ may not provide a stronger constraint on $\delta_{L L, 23}$. Since $\mathcal{O}_{7 \tilde{g}, \tilde{g}}$ requires a chirality flip within the loop, then at least an additional off-diagonal element different from zero is needed in the left-left sector of the down-squark mass matrix squared. Indeed, the flavor-conserving left-right mixing term $\left(m_{d, L R}^{2}\right)_{33}$ together with $\left(m_{d, L L}^{2}\right)_{23}$ can also generate the operator $\mathcal{O}_{7 \tilde{g}, \tilde{g}}$; see the first diagram in Fig. 3. The corresponding branching ratio is shown in Fig. 12, as a function of $\delta_{L L, 23}$ for different choices of $\delta_{L R, 33}$. The value of the diagonal entries in the squark mass matrix is $m_{\tilde{q}}=500 \mathrm{GeV}$ and $m_{g}$ is determined by the choice $x=0.3$. As in the previous plots, low and matching scales are fixed as $\mu_{b}=4.8 \mathrm{GeV}$ and $\mu_{W}=M_{W}$. Both parameters $\delta_{L R, 33}$ and $\delta_{L L, 23}$ are chosen to be positive. The solid line in this figure, obtained for $\delta_{L R, 33}$ $=0$, then coincides with the short-dashed line in Fig. 10. The

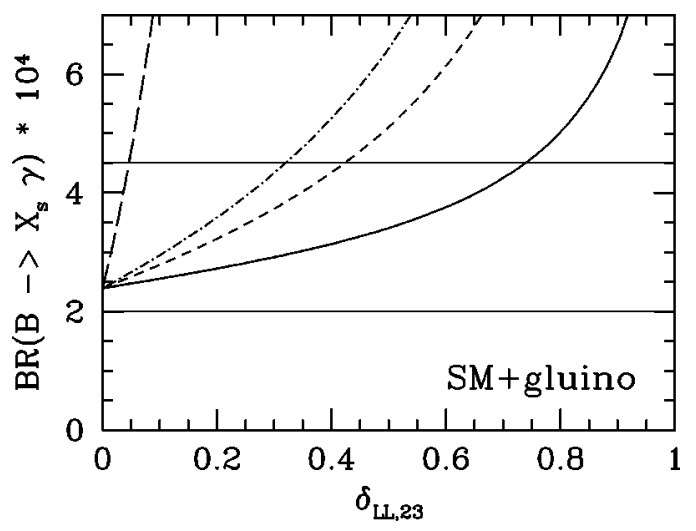

FIG. 12. $\operatorname{BR}\left(\bar{B} \rightarrow X_{s} \gamma\right)$ vs $\delta_{L L, 23}$, when $\delta_{L L, 23}$ and $\delta_{L R, 33}$ are the only sources of chiral-flavor violation. The dependence on $\delta_{L L, 23}$ is shown for different values of $\delta_{L R, 33}: 0$ (solid line), 0.006 (shortdashed line), 0.01 (dot-dashed line), 0.1 (long-dashed line). The value of $x=m_{\tilde{g}}^{2} / m_{\tilde{q}}^{2}$ is fixed at 0.3 and $m_{\tilde{q}}$ to $500 \mathrm{GeV}$.
SM value of the branching ratio, at the $\mathrm{LO}$ in $\mathrm{QCD}$, is the value at which all curves meet for $\delta_{L L, 23}=0$. The shortdashed line is obtained for $\left(m_{d, L R}^{2}\right)_{33} \simeq m_{\tilde{q}} m_{b}$, which corresponds to a relatively large trilinear coupling in models in which the trilinear term in the soft potential is proportional to the Yukawa couplings. The corresponding maximally allowed value of $\delta_{L L, 23}$ already is, in this case, considerably smaller than that obtained when only the operator $\mathcal{O}_{7 b, \tilde{g}}$ is present. Larger values of $\delta_{L R, 33}$ obviously induce even more stringent constraints on $\delta_{L L, 23}$.

Two obvious lessons can be learned out of this analysis. First, in directions of the supersymmetric parameter space in which other contributions to $\operatorname{BR}\left(\bar{B} \rightarrow X_{s} \gamma\right)$ cannot be neglected, some of the constraints derived here may be invalidated by possible interferences among different contributions. An illustration of this is provided by the comparison of the bounds imposed by $\mathrm{BR}\left(\bar{B} \rightarrow X_{s} \gamma\right)$ on $\delta_{L R, 23}$ and $\delta_{R L, 23}$, which are different precisely because contributions from SM-gluino interferences are possible in one case, but not in the other. The second lesson stems from the observation that different operators contributing to $\operatorname{BR}\left(\bar{B} \rightarrow X_{s} \gamma\right)$ have very different numerical relevance. Because of this, it is not necessarily true that the strongest constraint on a chiral-flavorviolating sfermion mass term can be derived from the operator that is generated by it in the most straightforward way. Therefore, one cannot but end this section by stressing again the importance of analyses as complete as possible, when attempting to use the $b \rightarrow s \gamma$ decay as a model-building tool, constraining the soft supersymmetry-breaking terms.

\section{SUMMARY}

Gluino-mediated contributions to FCNC processes are useful probes of chiral-flavor-violating soft breaking terms. They are in general cleaner than chargino contributions, which are sensitive also to the CKM matrix, responsible for flavor violation in the SM and in two Higgs doublet models (2HDMs). Since they come with a coupling $\alpha_{s}$, they are usually rather large. Whether they are indeed much larger than chargino contributions is a model-dependent issue.

The presence of the coupling $\alpha_{s}$ makes these contributions also particularly interesting for FCNC processes in which QCD corrections play as important a role as the purely electroweak contributions. Exemplary among these processes is the decay $b \rightarrow s \gamma$. A specific analysis of the implementation of QCD corrections for the gluino contribution to this decay is required. This paper is devoted to precisely this issue: it shows how to QCD-correct the gluino contribution to the decay $b \rightarrow s \gamma$, using the formalism of the effective Hamiltonian.

It is shown here that, contrary to the common belief, gluino contributions require an enlargement of the standard basis of operators needed to describe $b-s$ transitions in the SM and 2HDM's. In the SM, the calculation at the LO in QCD includes all terms of the type $\left[\alpha_{s} \log \left(M_{W} / m_{b}\right)\right]^{N}$, whereas the calculation at the NLO resums all terms $\alpha_{s}\left[\alpha_{s} \log \left(M_{W}^{2} / m_{b}^{2}\right)\right]^{N}$. The program of implementation of QCD corrections in the SM requires that at each order in 
QCD, e.g. LO or NLO, the anomalous-dimension matrix of the SM operators is calculated at a higher order in $\alpha_{s}$ than the matching conditions and the matrix elements. This is because in the SM, at a certain order in QCD, no other operator can mix into the magnetic operator $\left[e /\left(16 \pi^{2}\right)\right]\left(\bar{s} \sigma^{\mu \nu} P_{R} b\right) F_{\mu \nu}$ without the exchange of a virtual gluon. The situation is different in the case of gluino contributions. Gluino-induced magnetic operators acquire corrections as in the SM, when an additional virtual gluon is exchanged. Moreover, as in the SM, also gluino-induced chromomagnetic operators mix into the gluino-induced magnetic ones after the on-shell gluon is connected to a quark line and an additional photon is radiated. Both operators get first non-vanishing contributions at the matching scale at order $\alpha_{s}$, and give QCD-corrected contributions of type $\alpha_{s}^{2} \log \left(M_{W}^{2} / m_{b}^{2}\right)$. Gluino-induced four-quark operators with first non-vanishing contributions at the matching scale of $\mathcal{O}\left(\alpha_{s}^{2}\right)$, however, can mix into the magnetic operators through the connection of two of the external quark lines and the emission of an on-shell photon, giving therefore also corrections of type $\alpha_{s}^{2} \log \left(M_{W}^{2} / m_{b}^{2}\right)$. As not all logarithms are due to gluon exchange, their systematic resummation is more involved as in the SM.

A solution to this problem has been proposed in this paper. The couplings $\alpha_{s}$ and $\alpha_{s}^{2}$ intrinsically connected with the gluino exchange are respectively factorized out in the definition of magnetic and chromomagnetic operators and of operators originating from box diagrams. With this definition, all gluino-induced operators are distinguished from the standard set of operators in the effective Hamiltonian induced by SM and 2HDMs. In particular, the magnetic operator $\mathcal{O}_{7}=\left[\left(e /\left(16 \pi^{2}\right)\right] \bar{m}_{b}\left(\bar{s} \sigma^{\mu \nu} P_{R} b\right) F_{\mu \nu}\right.$ is now distinct from the gluino-induced one $\mathcal{O}_{7 b, \tilde{g}}$ $=e g_{s}^{2} \bar{m}_{b}\left(\bar{s} \sigma^{\mu \nu} P_{R} b\right) F_{\mu \nu}$. This in turn has to be distinguished from the lower dimensionality operator $\mathcal{O}_{7 \tilde{g}, \tilde{g}}$ $=e g_{s}^{2}\left(\bar{s} \sigma^{\mu \nu} P_{R} b\right) F_{\mu \nu}$, induced at the matching scale by a loop diagram in which chiral-flavor violation is provided, for example, by the insertion of a left-right mass term in the squark propagator and the insertion of a gluino mass in the gluino propagator. Completely new are the four-quark operators, such as $\mathcal{O}_{15, \tilde{g}}^{q}=g_{s}^{4}(\mu)\left(\bar{s} P_{L} b\right)\left(\bar{q} P_{L} q\right)$, with an explicit factor $g_{s}^{4}$. In total, the inclusion of gluino contributions requires 56 new operators and another additional 56 with opposite chirality.

With the above definition of gluino-induced operators, an important goal is achieved. The first non-vanishing contribution to the gluino-induced Wilson coefficients is of $\mathcal{O}\left(\alpha_{s}^{0}\right)$. Moreover, the anomalous dimension matrix starts at $\mathcal{O}\left(\alpha_{s}\right)$. Consequently, the integration of the renormalization group equation yields, at first non-vanishing order, terms all of the type $\left[\alpha_{s} \log \left(M_{W} / m_{b}\right)\right]^{N}$. The analogy with the LO SM contributions is now clear. It is this first non-vanishing order that is classified as LO gluino contributions. Thus, gluino exchanges induce terms of the type $\alpha_{s}\left[\alpha_{s} \log \left(M_{W} / m_{b}\right)\right]^{N}$ at the LO in QCD, to be compared to the LO SM contributions of the type $G_{F}\left[\alpha_{s} \log \left(M_{W} / m_{b}\right)\right]^{N}$. The generalization to the NLO is obvious: it will yield contributions $\alpha_{s}^{2}\left[\alpha_{s} \log \left(M_{W} / m_{b}\right)\right]^{N}$ for the gluino-induced operators, versus the contributions $G_{F} \alpha_{s}\left[\alpha_{s} \log \left(M_{W} / m_{b}\right)\right]^{N}$ coming from the SM set of operators.

A complete $\mathrm{LO}$ analysis for the branching ratio of the inclusive decay $\bar{B} \rightarrow X_{s} \gamma$ coming from SM and gluinoinduced contributions is presented in this paper. The full anomalous-dimension matrix for gluino-induced operators is calculated and a simple expression for the branching ratio is given. The gluino-induced Wilson coefficients are also listed. They are obtained from the evaluation of one-loop diagrams mediated by the exchange of gluino and squarks. The mass eigenstate formalism is adopted as the most suitable for supersymmetric models with different sources of flavor violation and with a priori large flavor-violating mass terms.

A numerical analysis for the inclusive branching ratio $\operatorname{BR}\left(\bar{B} \rightarrow X_{s} \gamma\right)$ due to SM and gluino-induced contributions is presented. The QCD corrections to the gluino-induced contributions are found to be even more crucial than in the SM case. The non-corrected contributions to the inclusive decay $\bar{B} \rightarrow X_{s} \gamma$, in fact, suffer from a severe source of uncertainty that has no counterpart in the SM. At the zeroth order in QCD, there is no prescription to fix the scale of the overall factor $\alpha_{s}^{2}$ in the final expression of the branching ratio, intrinsically due to gluino exchanges: it can range from the matching scale $\sim M_{W}$ to the low-scale $\sim m_{b}$. Once QCD corrections are added, the bulk of this ambiguity is removed: this factor of $\alpha_{s}^{2}$ has to be evaluated at a low scale of $\mathcal{O}\left(m_{b}\right)$, although the exact value of this scale remains unknown. A similar uncertainty is due to the fact that the matching scale is only known to be of $\mathcal{O}\left(M_{W}\right)$. Thus, the LO branching ratio still suffers from matching- and low-scale uncertainties similar in size to those in the SM results.

Finally, we conclude by recalling that this analysis is valid in particular directions of the supersymmetric parameter space, in which charged Higgs, chargino and neutralino contributions can be neglected. In spite of the still large theoretical error, it provides bounds on the different sources of flavor violation that are present in these directions of parameter space. Further studies are called for to include NLO contributions as well as all the remaining supersymmetric contributions.

\section{ACKNOWLEDGMENTS}

We thank G. Buchalla, P. Minkowski, and A. Pomarol for discussions. This work was partially supported by the Schweizerischer Nationalfonds and by the European Commission through the TMR Network under contract No. ERBFMX-CT960090. T.H. acknowledges financial support by the DOE under grant no DE-FG03-92-ER 40701 during a visit of the theory group at CALTECH where part of this work was done.

\section{APPENDIX A: FUNCTIONS}

Listed below are the loop functions appearing in the coefficients (29) and (30): 


$$
\begin{aligned}
& F_{1}(x)=\frac{1}{12(x-1)^{4}}\left(x^{3}-6 x^{2}+3 x+2+6 x \log x\right) \\
& F_{2}(x)=\frac{1}{12(x-1)^{4}}\left(2 x^{3}+3 x^{2}-6 x+1-6 x^{2} \log x\right) \\
& F_{3}(x)=\frac{1}{2(x-1)^{3}}\left(x^{2}-4 x+3+2 \log x\right), \\
& F_{4}(x)=\frac{1}{2(x-1)^{3}}\left(x^{2}-1-2 x \log x\right)
\end{aligned}
$$

those originated by the calculation of penguin diagrams [see coefficients (31)]:

$$
\begin{aligned}
F_{5}(x)= & \frac{1}{36(x-1)^{4}}\left[7 x^{3}-36 x^{2}+45 x-16\right. \\
& +(18 x-12) \log x]
\end{aligned}
$$

$$
\begin{aligned}
F_{6}(x)= & \frac{1}{36(x-1)^{4}}\left(-11 x^{3}+18 x^{2}-9 x+2\right. \\
& \left.+6 x^{3} \log x\right)
\end{aligned}
$$

and finally, the box-diagram functions:

$$
\begin{aligned}
& F(x, y)=-\frac{1}{x-y}\left[\frac{x \log x}{(x-1)^{2}}-\frac{1}{x-1}-(x \rightarrow y)\right], \\
& G(x, y)=\frac{1}{x-y}\left[\frac{x^{2} \log x}{(x-1)^{2}}-\frac{1}{x-1}-(x \rightarrow y)\right] .
\end{aligned}
$$

\section{APPENDIX B: WILSON COEFFICIENT REMAINDERS}

The effect of the four-quark operators (15) on the evolution of the Wilson coefficient relative to the magnetic and chromomagnetic operators $(11)-(13)$ is encoded in the remainder functions $R_{7 q, \tilde{g}}\left(\mu_{b}\right)$ and $R_{8 q, \tilde{g}}\left(\mu_{b}\right)(q=b, c)$ listed below:

$$
\begin{aligned}
& R_{7 b, \tilde{g}}\left(\mu_{b}\right)=\left(-\frac{2353}{33276}\left(d_{1}+d_{2}\right)+\frac{34105 \sqrt{241}}{8019516}\left(d_{1}-d_{2}\right)+\frac{100}{141} d_{3}-\frac{67}{118} d_{6}\right) C_{15, \tilde{g}}^{b}\left(\mu_{W}\right) \\
& +\left(-\frac{595}{33276}\left(d_{1}+d_{2}\right)-\frac{27749 \sqrt{241}}{8019516}\left(d_{1}-d_{2}\right)-\frac{32}{141} d_{3}+\frac{31}{118} d_{6}\right) C_{16, \tilde{g}}^{b}\left(\mu_{W}\right) \\
& +\left(+\frac{1181}{2773}\left(d_{1}+d_{2}\right)+\frac{7131 \sqrt{241}}{668293}\left(d_{1}-d_{2}\right)-\frac{48}{47} d_{3}+\frac{10}{59} d_{6}\right) C_{19, \tilde{g}}^{b}\left(\mu_{W}\right) \\
& +\left(+\frac{1767}{2773}\left(d_{1}+d_{2}\right)-\frac{13487 \sqrt{241}}{668293}\left(d_{1}-d_{2}\right)-\frac{224}{47} d_{3}+\frac{206}{59} d_{6}\right) C_{20, \tilde{g}}^{b}\left(\mu_{W}\right), \\
& R_{8 b, \tilde{g}}\left(\mu_{b}\right)=\left(+\frac{391 \sqrt{241}}{45308}\left(d_{1}-d_{2}\right)-\frac{25}{188}\left(d_{1}+d_{2}-2 d_{3}\right)\right) C_{15, \tilde{g}}^{b}\left(\mu_{W}\right) \\
& +\left(-\frac{20 \sqrt{241}}{11327}\left(d_{1}-d_{2}\right)+\frac{2}{47}\left(d_{1}+d_{2}-2 d_{3}\right)\right) C_{16, \tilde{g}}^{b}\left(\mu_{W}\right) \\
& +\left(-\frac{231 \sqrt{241}}{11327}\left(d_{1}-d_{2}\right)+\frac{9}{47}\left(d_{1}+d_{2}-2 d_{3}\right)\right) C_{19, \tilde{g}}^{b}\left(\mu_{W}\right) \\
& +\left(-\frac{702 \sqrt{241}}{11327}\left(d_{1}-d_{2}\right)+\frac{42}{47}\left(d_{1}+d_{2}-2 d_{3}\right)\right) C_{20, \tilde{g}}^{b}\left(\mu_{W}\right), \\
& R_{7 c, \tilde{g}}\left(\mu_{b}\right)=\left(-\frac{2375}{33276}\left(d_{1}+d_{2}\right)+\frac{39119 \sqrt{241}}{8019516}\left(d_{1}-d_{2}\right)+\frac{576}{2773} d_{3}\right. \\
& \left.-\frac{1273}{33276}\left(d_{4}+d_{5}\right)-\frac{25937 \sqrt{241}}{8019516}\left(d_{4}-d_{5}\right)+\frac{32}{2773} d_{6}\right) C_{15, \tilde{g}}^{c}\left(\mu_{W}\right) \\
& +\left(+\frac{1747}{33276}\left(d_{1}+d_{2}\right)+\frac{7205 \sqrt{241}}{8019516}\left(d_{1}-d_{2}\right)-\frac{2824}{8319} d_{3}-\frac{5267}{33276}\left(d_{4}+d_{5}\right)\right.
\end{aligned}
$$




$$
\begin{aligned}
& \left.-\frac{85147 \sqrt{241}}{8019516}\left(d_{4}-d_{5}\right)+\frac{1528}{2773} d_{6}\right) C_{16, \tilde{g}}^{c}\left(\mu_{W}\right) \\
& +\left(-\frac{373}{2773}\left(d_{1}+d_{2}\right)-\frac{17843 \sqrt{241}}{668293}\left(d_{1}-d_{2}\right)-\frac{4800}{2773} d_{3}-\frac{3087}{2773}\left(d_{4}+d_{5}\right)-\frac{48119 \sqrt{241}}{668293}\left(d_{4}-d_{5}\right)\right. \\
& \left.+\frac{11720}{2773} d_{6}\right) C_{19, \tilde{g}}^{c}\left(\mu_{W}\right)+\left(+\frac{1001}{2773}\left(d_{1}+d_{2}\right)-\frac{28481 \sqrt{241}}{668293}\left(d_{1}-d_{2}\right)\right. \\
& \left.-\frac{7360}{2773} d_{3}+\frac{907}{2773}\left(d_{4}+d_{5}\right)+\frac{11091 \sqrt{241}}{668293}\left(d_{4}-d_{5}\right)+\frac{3544}{2773} d_{6}\right) C_{20, \tilde{g}}^{c}\left(\mu_{W}\right), \\
R_{8 c, \tilde{g}}\left(\mu_{b}\right)=(- & \left.\frac{25}{376}\left(d_{1}+d_{2}\right)+\frac{391 \sqrt{241}}{90616}\left(d_{1}-d_{2}\right)+\frac{216}{2773} d_{3}+\frac{13}{472}\left(d_{4}+d_{5}\right)-\frac{73 \sqrt{241}}{113752}\left(d_{4}-d_{5}\right)\right) C_{15, \tilde{g}}^{c}\left(\mu_{W}\right) \\
+ & \left.+\frac{1}{47}\left(d_{1}+d_{2}\right)-\frac{10 \sqrt{241}}{11327}\left(d_{1}-d_{2}\right)-\frac{353}{2773} d_{3}+\frac{5}{118}\left(d_{4}+d_{5}\right)+\frac{20 \sqrt{241}}{14219}\left(d_{4}-d_{5}\right)\right) C_{16, \tilde{g}}^{c}\left(\mu_{W}\right) \\
+ & \left.+\frac{9}{94}\left(d_{1}+d_{2}\right)-\frac{231 \sqrt{241}}{22654}\left(d_{1}-d_{2}\right)-\frac{1800}{2773} d_{3}+\frac{27}{118}\left(d_{4}+d_{5}\right)+\frac{393 \sqrt{241}}{28438}\left(d_{4}-d_{5}\right)\right) C_{19, \tilde{g}}^{c}\left(\mu_{W}\right) \\
+ & \left.+\frac{21}{47}\left(d_{1}+d_{2}\right)-\frac{351 \sqrt{241}}{11327}\left(d_{1}-d_{2}\right)-\frac{2760}{2773} d_{3}+\frac{3}{59}\left(d_{4}+d_{5}\right)-\frac{153 \sqrt{241}}{14219}\left(d_{4}-d_{5}\right)\right) C_{20, \tilde{g}}^{c}\left(\mu_{W}\right), \quad(\mathrm{B} 4)
\end{aligned}
$$

where the factors $d_{1}-d_{6}$ are given by

$$
\begin{array}{ll}
d_{1}=\eta^{(47+\sqrt{241}) / 23}, & d_{2}=\eta^{(47-\sqrt{241}) / 23}, d_{3}=\eta^{37 / 23}, \\
d_{4}=\eta^{(29+\sqrt{241}) / 23}, & d_{5}=\eta^{(29-\sqrt{241}) / 23}, d_{6}=\eta^{39 / 23} .
\end{array}
$$

Notice that in Eqs. (B1),(B2) there is no dependence on $C_{17, \tilde{g}}^{b}\left(\mu_{W}\right)$ and $C_{18, \tilde{g}}^{b}\left(\mu_{W}\right)$, as there is no dependence on $C_{17, \tilde{g}}^{c}\left(\mu_{W}\right)$ and $C_{18, \tilde{g}}^{c}\left(\mu_{W}\right)$ in Eqs. (B3),(B4). By inspecting the two anomalous-dimension matrices in Eqs. (38) and (39), it is easy to see that the two operators $\mathcal{O}_{17, \tilde{g}}^{q}, \mathcal{O}_{18, \tilde{g}}^{q}$, do not mix with the remaining ones $\mathcal{O}_{15, \tilde{g}}^{q}, \mathcal{O}_{16, \tilde{g}}^{q}, \mathcal{O}_{19, \tilde{g}}^{q}, \mathcal{O}_{20, \tilde{g}}^{q}, \mathcal{O}_{7 q, \tilde{g}}, \mathcal{O}_{8 q, \tilde{g}}$ in either of the two cases, $q=b$ and $q=c$.

[1] A.H. Chamseddine, R. Arnowitt, and P. Nath, Phys. Rev. Lett. 49, 970 (1982); R. Barbieri, S. Ferrara, and C.A. Savoy, Phys. Lett. 119B, 343 (1982); L.J. Hall, J. Lykken, and S. Weinberg, Phys. Rev. D 27, 2359 (1983).

[2] M. Dine, W. Fischler, and M. Srednicki, Nucl. Phys. B189, 575 (1981); S. Dimopoulos and S. Raby, ibid. B192, 353 (1981); L. Alvarez-Gaumé, M. Claudson, and M. Wise, ibid. B207, 96 (1982); M. Dine and A.E. Nelson, Phys. Rev. D 48, 1277 (1993); M. Dine, A.E. Nelson, and Y. Shirman, ibid. 51, 1362 (1995); M. Dine, A.E. Nelson, Y. Nir, and Y. Shirman, ibid. 53, 2658 (1996).

[3] M. Leurer, Y. Nir, and N. Seiberg, Nucl. Phys. B398, 319 (1993); B420, 468 (1994).

[4] M. Dine, R.G. Leigh, and A. Kagan, Phys. Rev. D 48, 4269 (1993); S. Dimopoulos and G.F. Giudice, Phys. Lett. B 357, 573 (1995).

[5] A. Pomarol and D. Tommasini, Nucl. Phys. B466, 3 (1996).

[6] A.G. Cohen, D.B. Kaplan, and A.E. Nelson, Phys. Lett. B 388, 588 (1996).

[7] R. Barbieri, G. Dvali, and L.J. Hall, Phys. Lett. B 377, 76 (1996).
[8] F.M. Borzumati, M. Drees, and M. Nojiri, Phys. Rev. D 51, 341 (1995).

[9] T. Goto, Y. Okada, and Y. Shimizu, Phys. Rev. D 58, 094006 (1998).

[10] F. Borzumati, M. Olechowski, and S. Pokorski, Phys. Lett. B 349, 311 (1995).

[11] F.M. Borzumati, hep-ph/9702307.

[12] ALEPH Collaboration, R. Barate et al., Phys. Lett. B 429, 169 (1998).

[13] CLEO Collaboration, S. Ahmed et al., CLEO CONF 99-10, hep-ex/9908022.

[14] S. Bertolini, F. Borzumati, and A. Masiero, Phys. Rev. Lett. 59, 180 (1987); N.G. Deshpande, P. Lo, J. Trampetic, G. Eilam, and P. Singer, ibid. 59, 183 (1987).

[15] H. Politzer, Phys. Rep., Phys. Lett. 14C, 130 (1974); A.I. Vainshtein, V.I. Zakharov, V.A. Novikov, and M.A. Shifman, Nucl. Phys. B120, 120 (1977); Phys. Rev. D 18, 2583 (1978); 19, 2815(E) (1979).

[16] B. Grinstein, R. Springer, and M.B. Wise, Phys. Lett. B 202, 138 (1988); Nucl. Phys. B339, 269 (1990). 
[17] A.J. Buras, M. Jamin, M.E. Lautenbacher, and P.H. Weisz, Nucl. Phys. B370, 69 (1992); B375, 501 (1992) (Addendum); M. Ciuchini, E. Franco, G. Martinelli, L. Reina, and L. Silvestrini, Phys. Lett. B 316, 127 (1993); M. Ciuchini, E. Franco, L. Reina, and L. Silvestrini, Nucl. Phys. B421, 41 (1994); M. Ciuchini, E. Franco, G. Martinelli, and L. Reina, Phys. Lett. B 301, 263 (1993); 334, 137 (1994); Nucl. Phys. B415, 403 (1994); M. Misiak, ibid. B393, 23 (1993); B439, 461(E) (1995); G. Cella, G. Curci, G. Ricciardi, and A. Viceré, Phys. Lett. B 325, 227 (1994); Nucl. Phys. B431, 417 (1994); A.J. Buras, M. Misiak, M. Münz, and S. Pokorski, ibid. B424, 374 (1994).

[18] M. Misiak and M. Münz, Phys. Lett. B 344, 308 (1995).

[19] A. Ali and C. Greub, Z. Phys. C 49, 431 (1991); 60, 433 (1993); Phys. Lett. B 259, 182 (1991); 361, 146 (1995); N. Pott, Phys. Rev. D 54, 938 (1996).

[20] K. Chetyrkin, M. Misiak, and M. Münz, Phys. Lett. B 400, 206 (1997); Nucl. Phys. B518, 473 (1998).

[21] K. Adel and Y.P. Yao, Phys. Rev. D 49, 4945 (1994); C. Greub and T. Hurth, ibid. 56, 2934 (1997); A.J. Buras, A. Kwiatkowski, and N. Pott, Phys. Lett. B 414, 157 (1997); Nucl. Phys. B517, 353 (1998); M. Ciuchini, G. Degrassi, P. Gambino, and G.F. Giudice, ibid. B527, 21 (1998).

[22] C. Greub, T. Hurth, and D. Wyler, Phys. Lett. B 380, 385 (1996); Phys. Rev. D 54, 3350 (1996).

[23] F.M. Borzumati and C. Greub, Phys. Rev. D 58, 074004 (1998); 59, 057501 (1999); hep-ph/9810240.

[24] A. Falk, M. Luke, and M. Savage, Phys. Rev. D 49, 3367 (1994); I.I. Bigi, M. Shifman, N.G. Uraltsev, and A.I. Vainshtein, Phys. Rev. Lett. 71, 496 (1993); A.V. Manohar and M.B. Wise, Phys. Rev. D 49, 1310 (1994); A. Falk, M. Luke, and M. Savage, ibid. 53, 2491 (1996); M.B. Voloshin, Phys. Lett. B 397, 295 (1997); Z. Ligeti, L. Randall, and M.B. Wise, ibid.
402, 178 (1997); A.K. Grant, A.G. Morgan, S. Nussinov, and R.D. Peccei, Phys. Rev. D 56, 3151 (1997); G. Buchalla, G. Isidori, and S.J. Rey, Nucl. Phys. B511, 594 (1998).

[25] A.L. Kagan and M. Neubert, Eur. Phys. J. C 7, 5 (1999).

[26] S. Bertolini, F. Borzumati, A. Masiero, and G. Ridolfi, Nucl. Phys. B353, 591 (1991).

[27] S. Bertolini, F. Borzumati, and A. Masiero, Phys. Lett. B 192, 437 (1987).

[28] F. Gabbiani, E. Gabrielli, A. Masiero, and L. Silvestrini, Nucl. Phys. B477, 321 (1996).

[29] M. Ciuchini, G. Degrassi, P. Gambino, and G.F. Giudice, Nucl. Phys. B534, 3 (1998).

[30] C. Bobeth, M. Misiak, and J. Urban, Nucl. Phys. B567, 153 (2000).

[31] J.F. Donoghue, H.P. Nilles, and D. Wyler, Phys. Lett. 128B, 55 (1983).

[32] F. Borzumati, Z. Phys. C 63, 291 (1994).

[33] See for example, J.S. Hagelin, S. Kelley, and T. Tanaka, Nucl. Phys. B415, 293 (1994).

[34] M. Misiak, Nucl. Phys. B393, 23 (1993); B439, 461 (1995); A.J. Buras and M. Münz, Phys. Rev. D 52, 186 (1995).

[35] L.J. Hall, V.A. Kostelecki, and S. Raby, Nucl. Phys. B267, 415 (1986).

[36] F. Borzumati, G.R. Farrar, N. Polonsky, and S. Thomas, Nucl. Phys. B555, 53 (1999); hep-ph/9712428; hep-ph/9805314.

[37] J.M. Gerard, W. Grimus, A. Raychaudhuri, and G. Zoupanos, Phys. Lett. 140B, 349 (1984).

[38] M. Dougan, B. Grinstein, and L. Hall, Nucl. Phys. B255, 413 (1985).

[39] P. Cho, M. Misisak, and D. Wyler, Phys. Rev. D 54, 3329 (1996).

[40] G. Buchalla, A.J. Buras, and M.E. Lautenbacher, Rev. Mod. Phys. 68, 1125 (1996). 